\section{A bibliography experiment on research within the scope of industry 4.0 application areas in sports}

\section{Sporda endüstri 4.0 uygulama alanlar1 kapsaminda yapilan araştırmalar üzerine bir bibliyografya denemesi}

\author{
Y1lmaz Ünlü ${ }^{1}$ \\ Zeki Taş ${ }^{2}$
}

\begin{abstract}
Developed countries develop their production sites within the scope of industry 4.0 technology components and experience constant change and transformation to establish economic superiority. This situation allows them to produce more in various fields and thus to rise to a more advantageous position economically. Industry 4.0 technology affects areas within the scope of the sports industry such as sports tourism, athlete performance, athlete health, sports publishing, sports textile products, sports education and training, sports management and human resources, and creates an international competition environment in terms of production and performance. In this study, it is aimed to examine the researches about the usage areas of industry 4.0 in sports. From this point on, researches in the context of the subject have been presented with bibliographic method. In the conclusion section, the weaknesses and possibilities of youth sociology were discussed, and efforts were made to present a projection on what to do about the field. In this respect, a
\end{abstract}

Özet

Gelişmiş ülkeler endüstri 4.0 teknolojisi bileşenleri kapsamında üretim sahalarını geliştirmekte ve ekonomik üstünlük kurmak amaciyla sürekli değişim ve dönüşüm yaşamaktadır. Bu durum onların çeşitli alanlarda daha fazla üretmelerine dolayisiyla ekonomik yönden daha avantajlı konuma yükselmelerine olanak sağlamaktadır. Endüstri 4.0 teknolojisi spor turizmi, sporcu performans1, sporcu sağlığ1, spor yayıncilı̆̆ı, spor tekstil ürünleri, spor eğitimi ve öğretimi, spor yönetimi ve insan kaynakları gibi spor endüstrisi kapsamındaki alanları etkilemekte üretim ve performans yönünden ülkeler arası bir rekabet ortamı oluşturmaktadır. Bu çalışmada endüstri 4.0'in sporda kullanım alanları ile ilgili araştırmaların incelenmesi hedeflenmektedir. Bu noktadan hareketle konu bağlamındaki araştırmalar bibliyografik metodla ortaya konmuştur. Sonuç bölümünde ise sporda endüstri 4.0 kullanım alanları tartışılmıs, alana olan katkıları ve olumuz etkilerinin değerlendirilmesi yapılmıştır.

\footnotetext{
${ }^{1}$ Okutman, Bartın Üniversitesi, Spor Bilimleri Fakültesi, yunlu@bartin.edu.tr ID Orcid ID: 0000-0002-2190-3300

2 Okutman, Bartın Üniversitesi, Spor Bilimleri Fakültesi, zekitas54@gmail.com ID Orcid ID: 0000-0001-7781-5898
} 
Ünlü, Y., \& Taş, Z. (2020). Sporda endüstri 4.0 uygulama alanları kapsamında yapılan araştırmalar üzerine bir bibliyografya denemesi. Journal of Human Sciences, 17(4), 1149-1176. doi:10.14687/jhs.v17i4.6088

youth sociology evaluation has been tried to be made on the prominent topics, forgotten aspects and themes left incomplete in youth sociology studies.

Keywords: Industry 4.0, Sports, Bibliography.

(Extended English summary is at the end of this document)
Anahtar Kelimeler: Endüstri 4.0, Spor, Bibliyografya.

\section{Giriş}

Bilgi teknolojilerindeki gelişmeler akademik çalışmalarda bilgiye ulaşmayı her geçen gün daha da kolaylaştırmaktadır. Internet kullanımının ardından veri tabanlarına ulaşmayı kolaylaştıran programların geliştirilmesi ve bu gelişime bağlı olarak yaygınlaşması araştırmacılara özellikle zamanı verimli kullanma açısından büyük kolaylıklar sağlamaktadır. Araştırma konusuyla ilgili farklı veri tabanlarındaki çalışmalara ulaşmada büyük kolaylıklar sağlayacaktır. Bu yönüyle bibliyografik çalışmalar araştırmacı ile araştırılan konu arasında bir köprü vazifesi görmesi bakımından araştırmacıyı bilgi kaynağına yönlendirerek alanla ilgili çalışmalardan haberdar etme görevi görür.

Sporda teknoloji kullanımı, artan rekabete bağlı olarak performans gelişimi sağlamak spor sakatliklarının önüne geçmek ve vücudun farklı organlarına ait parametrelerin takibini istatistiki yönden değerlendirmek gibi amaçlarla sporun farklı alanlarında yaygınlaşmaktadır (James vd., 2016). Bu gelişme; sporcunun spor faaliyeti esnasında kullandığı kıyafetten, spor malzeme ve materyallerine, sporcu beslenmesine, spor faaliyetleri kapsamındaki dijital sistemlere, mobil uygulamalara, akıllı spor tesisleri ile entegrasyonuna ve performans ölçümlerinde kullanılan istatistiki hesaplamalara kadar geniş bir yelpazeyi kapsamaktadır. Sporda teknoloji kullanımı yoluyla spor aktivitelerinden ve spor organizasyonlarından beklenen performans artşı sporcu, antrenör, spor tesisi ve spor seyircisi gibi sporun bileşenlerine indirgenmektedir.

$\mathrm{Bu}$ çalışmada, sporda endüstri 4.0 kapsamında yapılan araştırmaları ortaya koymak amaçlanmıştır. Bu minvalde araştırma, konuyla ilgili asıl kaynağa ulaşmak isteyen araştırmacılara rehberlik görevi de görmektedir. Konu kapsamındaki bazı araştırmaların içeriğinden bilgiler verilmiş, araştırma kapsamındaki veri tabanlanı taranarak yapılan çalışmalar listelenmisstir. Tespit edilen araştırmalar; yazar soyadı ve adı, tarih, araştırma konusu, araştırmanın yayımlandığı bülten, cilt ve sayfa numaralarını kapsamaktadır.

\section{Genel Bilgiler}

\subsection{Tarihsel Süreçte Endüstri Devrimleri ve Endüstri 4.0 Kavramı}

Sanayi devrimlerinin tarihsel süreç içerisinde toplumlar üzerinde farklı ve derin etkiler bıraktı̆̆1 görülmektedir. Birinci endüstri devriminin tüm Avrupaya yayılmasının 120 yllı bulması, ikinci ve üçüncü sanayi devrimlerinin toplumsal etkilerinin nüfusun \%17 sini kapsaması, buna karşın Endüstri 4.0 içerisinde yer alan internetin 10 yıl gibi kısa zaman içinde tüm dünyaya yayıldığı görülmektedir (Schwab, 2019).

Endüstri devrimleri tarihine bakıldığında 18. yüzyılın sonuna doğru ilk olarak su ve buhar gücünün sanayide kullanılmasıyla birinci endüstri devrimi başlamıştır. 19. yüzyılın bitiminde elektrik enerjisinin sanayinin tüm alanlarında kullanılmaya başlanması ve böylece ilk seri üretimin başlamasıyla ikinci sanayi devrimi, 20. yüzyılın ortalarında elektronik ve bilgi teknolojilerinin gelişmesiyle de üçüncü sanayi devrimi süreci ortaya çıkmıstır.

Endüstri devrimleri sürecinde üretimin tarihsel sürecine bakıldığında ilk olarak zanaat olarak başlayan süreç daha sonra sırasıyla kitle üretimi, otomasyona dayalı üretim, müsteri odaklı ve kitle özelleştirilmiş üretim aşamaları şeklinde gerçekleşmiştir. Yine bu dönemde tüketicilerde en kaliteli 
Ünlü, Y., \& Taş, Z. (2020). Sporda endüstri 4.0 uygulama alanları kapsamında yapılan araştırmalar üzerine bir bibliyografya denemesi. Journal of Human Sciences, 17(4), 1149-1176. doi:10.14687/jhs.v17i4.6088

ürünü en ucuz fiyata alma eğilimi ortaya çıkmış daha sonra tüketiciler düşük fiyatlı kişiselleştirilmiş ürünleri tercih etmeye başlamışlardır. Bu durum, üretimde kitle özelleştirmenin de ötesine giderek ileri kişiselleşmiş ya da kişisel ürünler yaklaşımının ortaya çıkmasını sağlamıştır. Sonuç olarak ileri düzeyde kişiselleşmiş ürünler farklı iş uygulamaları ve iş döngülerini beraberinde getirmiştir ( Schumachera vd., 2016).

Sanayi 4.0 kavramı dördüncü endüstri devrimi için kullanılan bir terimdir (Drath ve Horc., 2016, ss.56-58). Endüstri 4.0 en kapsamlı tanımıyla, bireysel müşteri ihtiyaçlarını karşılamak amacıyla endüstriyel üretimi temsil eden, verimlilik, karll1ık ve kalite gibi hedeflerle, örgütlerde yönetim, ar-ge, üretim, pazarlama ve üretilen malların döngüsüne kadar geniş bir alanı etkileyen işletme üretim süreçlerinin dijitalleşmesidir (Neugebauer vd., 2016). Endüstri 4.0' in en belirgin özelliği işletmeyi meydana getiren iş parçalarını ve sistemleri birbirine bağlayarak sistemi oluşturan parçalar arasında akıllı ağlar oluşturarak kontrol mekanizmasını sağlamasıdır (Mueller vd., 2017, ss.1050-1057).

\subsection{Endüstri 4.0'1n Teknolojik Bileşenleri}

\subsubsection{Siber Fiziksel Sistemler (SFS)}

Siber fizksel sistemeler "siber" ya da "sibernetik" olarak bilinen ve canlı varliklar ile makineler arasında geçen etkileşim ve kontrolü, araştırma konusu edinmiş bilimsel bir disiplinden ortaya çıkmıştır (Bradley ve Atkins, 2015, ss. 23020-23049). Fiziksel dünya ile sanal dünyayı birbirine entegre eden bu sistem, fiziksel üretimlerin bilgisayar ortamında modellenmesi ve üretim sistemlerinin sanal ortamda da görülmesine olanak sağlamaktadır (Hermann vd. 2016). Diğer bir ifade ile internet tabanlı verilere erişim ve bu verileri işleme hizmetini eş zamanlı bir şekilde kullanarak fiziksel dünya ile yoğun etkileşim içinde nesnelerle işbirliği içinde olmaktır. Siber fiziksel sistemler, fiziksel dünya ile etkileşim kurma imkânı açısından ve geleceğin teknolojik gelişmeleri açısından önemli bir role sahiptir (Baheti, 2011, ss.161-166). Geliştirilen siber fiziksel sistemler, seçkin sporcuların performanslarının belirlenen özelliklere göre izlenmesine olanak sağlar ayrıca spor alanında güvenlik açısından kritik etki alanı için oldukça etkili çözümler sunmak için büyük bir potansiyel sağlar (McCaffery, 2018).

\subsubsection{Nesnelerin İnterneti (IOT)}

Nesnelerin interneti, insan müdahalesi olmadan nesnelerin diğer nesnelerle bağlantı kurarak özerk bir şekilde çalışmasıdır (Conti vd., 2018). Endüstriyel internet olarakta adlandırılan nesnelerin interneti, akıllı fabrikalar, akıllı ürünler ve akıllı servislerin temelini oluşturmaktadır (Kagermann, Lukas ve Wahlster 2011, s13). Diğer bir ifade ile kablolu ve kablosuz bağlantılarla ve benzersiz adresleme şemaları vasıtasıyla fiziksel dünyanın bilgisayar sistemleri ile etkileşim kurabilmesini sağlayan bir ağ sistemini temsil etmektedir. Nesnelerin internetinin amac1, herhangi bir hizmet ağını her zaman, her yerde, herhangi bir yolla kullanarak ağı kullanan herkesle, herhangi bir zaman ve mekânda etkileşim içinde olmayı sağlar (Vermesan ve Friess 2013). Nesnelerin interneti, spor sektöründe giyilebilir spor teçhizatları ve sensörler kullanılarak birçok araştırmada kritik veriler elde etmede kullanılmaktadır. Bu araştırmaların çerçevesi, hareket bilgileri (hız, hızlanma, konum vb), yaşamsal belirti bilgileri (kan şekeri, kan basıncı, vücut sıcaklığı, kalp atış hızı vb), çevresel bilgiler (sicaklık nem vb) şeklindedir (Al-Makhadmeh ve Tolba 2019, s. 106815).

\subsubsection{Yapay Zekâ}

Luger, (2009)'da yapay zekâyı akıllı davranışların makineler vasıtası ile kendiliğinden yapılmasını sağlamak şeklinde tanımlamıştır. Özetle insan beyninin sahip olduğu özelliklerin belirli yöntemlerle makinelere aktarılmasıdır (Makridakis, 2017, s.46-90). Çalışmasında yapay zekânın toplum her yönüyle etkileyeceği, artan küresel rekabetten dolayı daha yaygın hale gelen yapay zekâ kullanımının, hem toplum üzerinde hem de işletmeler üzerinde bazı avantaj ve dezavantajları olduğunu belirtmiştir.

Yapay zekâ, spor olumlu anlamda etkilemekte bireysel ve takım performansını yükseltmede etkin bir role sahip olduğu görülmektedir. Özellikle tenis, futbol, beyzbol gibi birçok branşta kullanılan yapay zekâ, rakip oyuncu ve takımlar hakkında verilerin elde edilmesinde, sporculara, televizyon yayıncılarına, spor seyircilerine sürekli olarak oyun içgörüsü kazandırmada etkisi oldukça fazladır. Oyuncunun oyun esnasında doğru prosedürü seçmesi, tehlike anında oyuncuyu alarma 
Ünlü, Y., \& Taş, Z. (2020). Sporda endüstri 4.0 uygulama alanları kapsamında yapılan araştırmalar üzerine bir bibliyografya denemesi. Journal of Human Sciences, 17(4), 1149-1176. doi:10.14687/jhs.v17i4.6088

geçirecek stratejileri tahmin etmesi ile spor yaralanmalarının azalmasına katkı sağlamaktadır (Nadikattu 2020, s.5983-5997).

\subsubsection{Büyük Veri}

Big data, çeşitli yapılandırılmıs, yarı yapılandırılmış ve yapılandırılmamış veriler ve bu verilerin birleşimi ile farklı veri formatları şeklinde tanımlanmaktadır. Veri formatı ile metin mesajlanı, belge, eposta, görüntü, ses, video, grafik verileri gibi verilerden bahsedilmektedir (Shaw vd. 2016, ss.347-385). İş zekâsı, iş analitiği ve büyük veri analitiği alanı son yirmi yıl içerisinde hem akademik hem de iş çevresinde giderek önemli hale gelmeye başlamışır. Sektör araştırmaları, bu durumun önemli bir gelişme olduğunu vurgulamıştır. Big data teknolojisi kuruluşlara veri analizi ile ilgili firsatlar sunarak, kritik iş verilerinin çözümünde ve kararlar almada önemli bir destek sunmaktadır (Chen, Chiang, ve Storey 2012, ss.1165-1188).

Spor; oyucular, takımlar, seyirciler ile ilgili büyük miktarda veri üretmektedir. Büyük veri sayesinde bu verilerin büyük miktarını analiz etmek ve kullanmak mümkün hale gelmektedir. Oyuncuların kendilerini analiz edebilmelerine, antrenörlerin doğru oyucu seçebilmelerine, hakemlerin doğru kararlar verebilmesine ve spor seyircilerininin oyuna çekilmesine olanak tanımaktadır. Günümüzde spor istatistiklerine talep oldukça fazla olduğundan spor sektörü için büyük verinin önemi giderek artmaktadır (Dmonte ve Dmello 2017).

\subsubsection{Bulut Bilişim}

Bulut bilişim, veri ve uygulamaları desteklemek için internet ve merkezi kontrol hizmetini kullanan internet odaklı teknolojik bir sistemdir. Tüketicilerin ve işletmelerin kişisel dosyalarına bilgisayar ve interenet aracıllğı ile onlara yaklaşmadan erişimini ifade eden teknolojidir (Padmapriya, Subhasri, ve Engineering 2013, ss.3). Bulut bilişim tüketici ve kuruluşlara sermaye tasarrufu ve işletme tasarrufu açısından çeşitli faydalar sağlamaktadır. Bu faydalara rağmen bulut bilişimin getirdiği bazı kısıtlamalar da mevcuttur. Güvenlik bu kısttların en önemlisidir. Güvenlik sorunu kişi ve kurumlar açısından kişisel, etik ve finansal zararlara neden olabilmektedir (Subramanian, Jeyaraj, ve Engineering 2018, 28-42).

\subsubsection{Arttırılmış Gerçeklik}

Artırılmış gerçeklik ses, video, grafikler veya konum verileri gibi bilgisayar tarafindan üretilen unsurlarla birleştirilmiş gerçek dünyanın bir görünümünü içerir (Sourin 2017, ss.49-64). Sanal gerçeklik gerçek dünyayı bilgisayar tarafindan üretilen öğelerle değiştirirken, kullanıcıların çevredeki çevreye ilişkin algısını geliştirmek için fiziksel dünya ile birlikte çalışır (Wiederhold, 2019). Arttırılmış gerçeklik, fiziksel dünyanın onunla ilgili dijital bilgilerle entegre olmasını ifade etmektedir. İş firsatları açısından arttırrlmış gerçeklik uygulamaları günümüzde tüketicilerin çalışma ve alışveriş şekillerini değiştirmek için kullanılabilmektedir (Farshid vd. 2018, ss.459). Artırılmış gerçeklik futbol, kriket, beyzbol, tenis gibi branşlarda oyunlarda kullanılan topun gitmekte olduğu yolu takip etmek amaciyla kullanılmaktadır (İçten ve Güngör 2017, ss.111-136).

\subsection{Sporda Endüstri 4.0 Kapsamındaki Araştırmalara Genel Bir Bakı̧̧}

\subsubsection{Giyilebilir Ak1lli Cihazlar}

Sporda endüstri 4.0 kapsamında kullanılan giyilebilir teknoloji ürünlerinde daha çok sensör özellikli kıyafetler öne çıkmaktadır. "Sanal sensör" olarak bilinen bu sistem sayesinde başta spor sektörü olmak üzere eğlence ve sağlık sektörlerinde de deney içerikli birçok çalışmanın varlığ1 görülmektedir. Özellikle atletlerin daha fazla istifade edebileceği bir çalışmada Caporaso ve arkadaşları (T. Caporaso vd., 2020) belirli biomekanik parametrelere dayanan giyilebilir bir cihaz tasarlamışlardır. "Atalet sistemi" adı verilen bu cihaz yardımıla yürüme esnasında sporcunun pasif ve hatalı hareketlerinin ortaya çıkarılması hedeflenmektedir. Mobil bir cihaz yardımıyla antrenörün sporcuyu takip edebildiği bu sistemle yürüme esnasındaki aksayan yönlerin tespit edilip giderilmesi sağlanmakta ve sporcunun bu yöndeki performansında artışın sağlanması hedeflenmektedir. Statik ve dinamik koşullar altında diz hareketlerini izlemek için giyilebilir bir sistemin uygulamaya konulduğu bir çalışmada (Resta vd., 2020) sporcuların performanslarını geliştirmek için önemli bilgiler sağlayan sensörlü bir dizlik tasarlanmıştır. İnsan yürüyüşü, teşhis, izleme, rehabilitasyon ve spor dahil olmak 
Ünlü, Y., \& Taş, Z. (2020). Sporda endüstri 4.0 uygulama alanları kapsamında yapılan araştırmalar üzerine bir bibliyografya denemesi. Journal of Human Sciences, 17(4), 1149-1176. doi:10.14687/jhs.v17i4.6088

üzere çeşitli uygulamalarda kullanılan önemli bir ekipmandır. Eklem hareketleri esnasında diz fleksiyonu/ekstansiyonu çok önemli bir harekettir ve değerlendirilmesi patolojiler, yürüme bozuklukları, reçete edilen tedavilerin etkinliğini ve hastaların rehabilitasyon durumunu ortaya koyması bakımından önem arzetmektedir. Dolayısıyla sporda giyilebilir teknolojide sensör kullanımı en yaygin uygulamalar arasindadır.

Sporcu için, giyilebilir akıllı cihazlar aracilığıyla sağlık verilerinin toplandığı, Io'T destekli cihazlar ve sistemler spor ortamlarnnda kullanılmaktadır. Sporcunun her bir bireysel faaliyeti, giyilebilir cihazlardan toplanan verilerle tamamen izlenebilmektedir. Sporcunun koşma, düşme, yürüme, eğilme, yatma, turmanma gibi çeşitli aktiviteleri sırasında kalp atış hızı, kan basıncı, glikoz seviyesi ve oksijen seviyesi gibi sağlık faktörleri eksiksiz analiz edilebilmektedir. Ek olarak, sporcunun sağlık koşulları, aktif oyun süreleri boyunca duyarlı semptomlar için izlenerek analiz edilebilmektedir. Tüm bu veriler, veritabanı merkezleri veya bulut platformları gibi depolama yollarıla, daha fazla işlem ve analiz için sensör ağından toplanan verilerin depolanmasına yardımcı olmaktadır (Subasi vd., 2018).

Bisiklet egzersizi sırasında solunum izleme yoluyla performansın değerlendirildiği bir çalışmada (Massaroni vd., 2020) içerisine 12 fiber optik sensörün yerleştirildiği vücudun 12 farklı bölgesinden ölçüm yapabilen spor tipi akıllı bir tişört kullanılmıştır. Bisiklet sporu için giyilebilir spor solunum cihazlarının tasarımına ilişkin bilgileri sağlamak için böyle bir metodolojik yaklaşımı kullanmaya yönelik ilk girişimin olduğu ifade edilen çalışmada bisiklete binme sırasında ellerin gidona sabitlenmesi, gövdenin eğimli olması ve gögüs kaslarının gövdeyi stabilize etmek için aktif hale getirilmesi ve böylece göğüs kafesinin solunumla ilgili hareketlerini etkilemesi gibi hususlara dair ölçümler ve sonuçlar elde edilmiştir. Yüksek yoğunluklu egzersizler sırasında solunum ile ilgili parametrelerin elde edilmesi amacıyla yapılan başka bir çalışmada (Smyth, Winter, ve Dickinson 2020) Optoelektronik pletismografi (OEP)'nin egzersiz sirasinda disfonksiyonel solunum paterni olan ve olmayan sporcular arasında farklılık gösterip göstermediği araştırılmıstır. Araştırma bir solunum fonksiyonu ölçüm sistemi olarak OEP'nin yanı sıra potansiyel bir solunum tanı ve yönetim aracı olarak gelecekteki araştırmalar için bir temel sağlayacak niteliktedir.

Koşu performansının iyileştirilmesi amacıyla vücut hidrasyon analizinin yapıldığ1 bir çalışmada Lapadula ve arkadaşları (Lapadula vd., 2020) sporcuların maraton ve bisiklet gibi yoğun bir aktivite sırasında birkaç faktörün etkisiyle vücut hidrasyonuna bağlı performans düşüşünün nedenlerini araştırmıştır. Koşu esnasında kullanılan bir sensör yardımıyla hız, kalp atış hızı, yükseklik, kalori, mesafe, süre, adım sayısı, adım uzunluğu, adım frekansı ve adım gücü parametreleri tespit edilmiştir. Sporculardan alınan tükürük numunesinin laboratuvar ortamında incelenmesi ve sonuçların karşılaştırılması ile yapılan ölçüm sonuçları neticesinde vücut hidrasyonu ile yapılan spor yoğunluğu arasındaki ilişkinin boyutları ortaya konulmaya çalışılmıştır.

\subsubsection{Spor Sakatlıkları ve Sporcu Sağlığ1}

Spor eğitiminin daha verimli hale getirilmesi ve aktivite esnasında ortaya çıabilecek sakatlkkların en aza indirilmesi amacıyla sporda teknoloji kullanımı gittikçe artmaktadır. Sporcunun anlık durumunu hatta rakibin zaaflarını tespit etmek maksadıyla ölçüm yaparak veri elde eden ve bu veriyi kısa sürede analiz eden cihaz ve uygulamaların sayısında artış görülmektedir. Antrenörler sensör, kamera ve mobil uygulamalar aracıllğıyla sporcularını daha nitelikli takip edebilmekte ve böylece daha kaliteli yönetebilmektedir. Spor kıyafetine veya ayakkabısına yerleştirilen cihazlar spor aktiviteleri esnasında antrenöre anlık bilgiler verebilmekte karşılaşılan sakatlanma durumlarının nedenleri ve iyileşme süresini hızlandırmaya yönelik tedbirler için daha kesin çıkarımlar yapılabilmektedir.

Özellikle elit ve profesyonel sporlarda yaralanmaların önlenmesi iddialı ve zor bir hedeftir. Bunu başarmanın bir yolu, kas hasarının biyolojik belirteçlerinin erken saptanmasıdır (Dei vd., 2019). Kreatin kinaz ve interlökin-6 (IL-6) gibi sitokinler bu tür belirteçlerdendir (Toft vd., 2002, ss. c289-c295). Vücut enerjisi için önemli enzimlerden biri olan kreatin kinaz (CK) farklı hücre ve dokularda üretilir. CK hücrelerin doğru bir biçimde çalışmasında görevi bulunan bir protein şeklinde de tanımlanır. Kalp krizi belirtileri görülen vaziyetlerde, kas ve beyin hasarı şeklinde hastalıklarda teşhis koymak için kreatin kinaz yüksekliği testi yapılmaktadır. Buna karşı (Marques-Deak vd., 2006, ss.99-109) kreatin kinaz terde gösterilmezken, IL-6'nın kana benzer konsantrasyon seviyelerinde terde 
Ünlü, Y., \& Taş, Z. (2020). Sporda endüstri 4.0 uygulama alanları kapsamında yapılan araştırmalar üzerine bir bibliyografya denemesi. Journal of Human Sciences, 17(4), 1149-1176. doi:10.14687/jhs.v17i4.6088

göründüğ̈u bildirilmiştir. Dolayısıyla erken teşhis edilebilecek bu biyolojik belirteçlerin spor sakatlıklarının önüne geçmede hayati öneme sahip olduğu söylenebilir.

IoT'nin tıbbi alanda uygulanması, geleneksel tıbbi hizmet modunu değiştirmiştir. Io'T'ye dayalı spor yaralanmalarının tubbi teşhis sistemi, hastalar için uygun, hızlı ve uzaktan izleme imkânı sağlamıştır. Böylece sakatlığı kontrol etme veya hasarın ilerlemesine ilişkin süreci ortadan kaldırmaktadır. Böylece sporcuların hastane ve tıbbi masraflardan tasarruf etme imkânı ortaya çıkmaktadır. Io'T'ye dayalı spor yaralanmalarının tıbbi teşhis sistemi, kullanıcılar için çok çeşitli kategorilerde uzaktan mobil izleme gerçekleștirme sağlayabilmektedir. Ayrıca bu teknoloji gömülü teknoloji, bluetooth kablosuz iletişim teknolojisi ve mobil el terminali depolama teknolojisini birleştirerek hizmet sağlayan bir altyapıya sahiptir (Yu 2020, ss.e3929).

Sporcuların son zamanlarda sıklıkla giyme tercihinde bulunduğu ve endustri 4.0 kapsamında üretilen spor sütyenleri de spor sakatlıklarını engellemede önemli katkılar sağlamaktadır. Spor sütyenleri aşır1 yüklenme, istemsiz gögüs hareketleri, aktivite esnasında oluşabilecek gerilme ve zorlanmalara karşı üst ekstremite sakatlıklarını önleyebilmektedir. Böylece sporcunun kendinden beklenen harekete daha iyi odaklanması sağlanmakta ve performansına olumlu katkılar sağlamaktadır. Spor sütyenlerinin Endüstri 4.0 teknolojisiyle üretilmesindeki avantajlara değinilen bir çalışmada (Goes vd., 2020, ss.1-16) geleneksel üretime kıyasla üretim süresinde, işçilik maliyetinde enerji ve hammadde tüketiminde azalmalar kullanımda ise konfor ve bakım kolaylığ sağladığı ortaya konulmuştur.

Io'T ve analitik yaklaşımlanı kullanarak spor yaralanma risklerinin azaltılmasına yönelik yapılan diğer bir çalışmada (Wilkerson, Gupta ve Colston 2018, ss.1348-1360) sporcuların güvenliğinin ve refahının teşvik edilmesi için kesin sonuçlar üretebilen bulgulara ulaşılmıştır. Çalışma, birçok mesleki ortamda yüksek kas-iskelet sistemi yaralanmaları insidansını ele almak için uygulanabilmektedir. IoT cihazları kullanılarak toplananlar da dâhil olmak üzere farklı verilerin analizi, yüksek yaralanma riskine sahip sporcuları açıkça belirleyebilmektedir. Çeşitli metodolojik yaklaşımları kullanarak oyun maruziyetleri temelinde yaralanma oluşumunu tahmin etmek için ikili risk kategorizasyon modelinin kullanımını destekleyen kanıtlar, önceki yaralanmanın kendi kendine bildirilen kalıcı etkileri ve tek bacaklı postüral stabilitenin etkili bir ölçüsü sağlanabilmektedir

\subsubsection{Spor Malzeme ve Materyalleri}

Bilişim teknolojilerindeki teknolojik gelişmelerle birlikte spor endüstrisi, küresel olarak milyarlarca insana ulaşan ve onları etkileyen önemli bir eğlence endüstrisi haline gelmiştir. Bununla birlikte, spor endüstrisinin emniyetini ve sürdürülebilirliğini sağlamak ve ilerletmek için, kullanılan ürünlerin maliyeti, verimliliği, dayanıklıllğı, yeniden kullanılabilirliği ve geri dönüştürülebilirliğini sağlamak için üretim ve malzeme süreçlerinde her zaman teknolojik yeniliklere ihtiyaç vardır ( $\mathrm{Al}$ Rashid vd., 2020).

Spor uygulamaları için oksetik malzemelerin incelenmesi: konfor ve korumada genişletme seçenekleri adlı çalışmada (Duncan vd., 2018, ss.941) spor faaliyetleri kapsamında kullanılan yardımcı malzemeler incelenmiştir. Araştırma ağırlıklı olarak yardımcı köpükler, tekstiller ve spor faaliyeti esnasında oluşabilecek darbelere karşı koruma sağlayan ürünler üzerinedir. Araştırmada Oksetik malzemelerin çarpma kuvvetine veya ivmeyi zayıflatan senaryolara yardımcı olan benzersiz yüksek girinti direnci ve kırılma tokluğu sağlayabileceği belirtilmiştir. Bu tür malzemelerin spor kıyafetlerinde ve kişisel koruyucu ekipmanlarda rahatlı̆̆1, oturuşu ve dayanıklllı̆̆1 artırabileceği, çok eksenli genişleme, filtrasyon uygulamalarında da yararlı olabileceği, kayak, snowboard, tenis raketleri veya hokey sopalarının kütlesini azaltılabileceği bildirilmiştir.

Endüstri 4.0 kapsamındaki araştırmalar spor malzeme ve materyallerinde yaşanan büyük değişim ve dönüşümü ortaya koymaktadır. Üretilen spor malzemesinin kullanımının, kalitesinin ve seri üretiminin iyileştirilmesi yapılan aktiviteye katkı sağlamaktadır. Bu kapsamda Ali ve arkadaşlarının yaptığ bir çalışmada (Ali, Choudhary, ve Abbas 2018, ss.218-222) endüstri 4.0 konsepti altında farklı ünitelerden oluşan bir futbol topu üretim makinası imal edilmiştir. Bu makine ile üretilen futbol toplarının, kullanımda olan diğer futbol toplarına kıyasla daha kaliteli, daha az hataya sahip ve daha uzun süre kullanıma sahip olduğu ortaya konulmuştur. 
Ünlü, Y., \& Taş, Z. (2020). Sporda endüstri 4.0 uygulama alanları kapsamında yapılan araştırmalar üzerine bir bibliyografya denemesi. Journal of Human Sciences, 17(4), 1149-1176. doi:10.14687/jhs.v17i4.6088

\subsubsection{Tahmin Tabanlı Program veya Uygulamalar}

Spor veri madenciliği, antrenörlere ve yöneticilere sonuç tahmini, oyuncu performans değerlendirmesi, oyuncu sakatllğı tahmini, sporda yetenek belirleme ve oyun stratejisi değerlendirmesinde yardımc olur (Keshtkar Langaroudi, Yamaghani, ve Technology 2019, ss.27-36). Spor faaliyetlerine ait sonuçların tahmin edilmesine dayanan yapay zekâ tekniklerini içeren teknolojiler sporda endüstri 4.0 kapsamında önde gelen gelişmeler arasındadır. Fialho ve arkadaşları (Fialho, Manhães ve Teixeira 2019, ss.131-136) yapay sinir ağlarıla birbirine bağlı nöronlar aracıllğıyla bir takım algoritmalar eşliğinde spor müsabaka sonuçlarına ilişkin tahminlerde bulunan bir uygulama üretmişlerdir. Uygulama ile yapılan eşleşme sonuçlarına göre \% 63 oranında doğru tahmin sonucu ortaya çıkmıştır. Turnuva verilerinin kullanıldığı bir çalışmada (Rotshtein vd., 2005, ss. 619-630) çevrimdışı ve çevrimiçi optimizasyon algoritma tekniklerinden oluşturulan uygulama müsabaka sonuçlarının yüksek bir oranda doğru tahmin edilebilmesine ve ticari olarak kullanılmasına imkan tanımaktadır.

Veri madenciliği araçlanının kullanılması, maç öznitelikleri ile sonuçlar arasındaki ilişkinin ve performans değişkenlerine dayalı olarak sonuçların nasıl tahmin edileceğini veya optimize edileceğini derinlemesine anlamak için yeterli görülmemektedir. Hassan ve arkadaşları (Hassan vd., 2020) galibiyet, mağlubiyet ve niteliklerin hassasiyetlerini tahmin etmek için farklı bir yaklaşım önerdikleri araştırmada maç sonuçlarının tahminini mümkün kılan bir çalışma ortaya koymuştur. Radyal temel işlevli bir sinir ağı modeli olan bu çalışmada tüm maç özelliklerinin etkinliğine ilişkin veriler başarıyla elde edilmiş ve müsabaka sonuçlarını katılımcı gruplara galibiyet veya mağlubiyet olarak sınıflandırdığını ortaya koymuştur.

Sporcunun birbirini izleyen günlerdeki davranışlarını (örneğin beslenme, aktivite, uyku) tanımlayan ve fitness ile ilgili ölçümleri izleyen çalışmasında Barricelli ve arkadaşları (Barricelli vd., 2020) Dijital İkiz (DTs) adı verilen bir uygulama tasarlamışlardır. Yeterli ölçümleri topladıktan sonra, DTs ilk olarak antrenman sirasında fiziksel ikiz performansını tahmin eder ve optimal olmayan bir sonuç olması durumunda, sporcunun davranışında değişiklikler önermektedir. Sporcunun ekibi, sporcuların fitness aktivitelerini ve sonuçlarını izleme ve yönetmede antrenörleri destekleyen bir yazılım çerçevesi olan SmartFit'e entegre edilmiştir. SmartFit, giyilebilir cihazlara gömülü IoT sensörleri ve manuel günlük kayıt (örneğin ruh hali, yiyecek miktarı vb.) için uygulamalar aracilı̆̆ıyla, başlangiçta mevcut fiziksel ikizlerin durumunu açıklayan dinamik veriler olarak değerlendirilen ölçümleri sürekli olarak yakalamaktadır. Dinamik veriler, her DTs'nin durumunun uyarlanmasına ve DTs'nin tahmin ve önerilerinin tetiklenmesine izin verir. Analiz edilen ölçümler geçmiş veriler olarak saklanır ve DTs tarafından bilgi ve güvenilir tahminler sağlama becerisini güncellemek (artırmak) için daha fazla işlenir. Çalışmanın sonucunda DTs ekibi sayesinde, SmartFit'in fiziksel ikizlerin koşullarının güvenilir tahminlerini hesapladığını ortaya koymuştur. Sporcuların davranışlarında optimizasyon eylemlerini tetiklemek için eğitmenler tarafindan kullanılabilecek anlaşılır öneriler üretildiği tespit edilmiştir.

Itoh ve arkadaşlan hareket halindeki bir tenis topunu gerçek zamanlı olarak takip edebilen, gelecekteki yörüngesini tahmin edebilen ve onu kullanıcının görüş alanında görselleştirebilen bir sistem tasarlamışlardır (Itoh vd., 2016). Laplacian Vision (LV) adı verilen sistemde bir tenis müsabakası esnasında kaçırılan servis atışlarının ve topla raketi temas ettirmedeki hataların en aza indirilmesi amaçlanmıştır. Sistem kullanılarak yapılan ölçümler sporcunun bir topun iniş noktasına kadar olan takibini kolaylaştırdığı gibi tahminini de 3 kat arttı̆ını ortaya koymaktadırBu alanda çalışmak isteyen araştırmacıların bilimsel çalışmalarında kullanabilecekleri nicel bir ölçeğe ihtiyaç olduğu görülmüştür.

\subsubsection{Performans ve Analiz}

Son zamanlarda, profesyonel spor takımları organizasyonlan kendi ve rakiplerinin performansını analiz etmek için öz kaynaklarına yatırım yapmaktadırlar. Bu nedenle, takım sporlarını analiz etmek için yöntemler ve algoritmalar geliştirmek, veri bilimcileri arasında en popüler konulardan biri haline gelmiştir. Futbolu analiz etmek; karmaşıklığı, her maçtaki olay sayısı ve topun sürekli dolaşım akışı nedeniyle zordur. Bir takımın performansını analiz etmek veya oyuncular 
arasında anlamlı bir karşılaştırma yapmak amacıyla oyuncuların oyun esnasındaki rollerini bulmak önemlidir. Bu kapsamda Behrevan ve arkadaşlarının yaptı̆̆ bir çalışmada (Behravan vd., 2019, ss.3536) sürü istihbarat algoritmasına dayalı otomatik bir büyük veri kümeleme programı oluşturulmuştur. Program, farklı müsabakalarda oyuncuların performanslarına ait verileri otomatik olarak kümelemek ve futbolda farklı rollere ait görevleri tespit etmek için önerilmektedir.

Son teknolojik yenilikler nedeniyle, konum izleme verilerini toplayan ve sağlayan sistem ve cihazlarda belirli bir artış olmuştur. Bu yenilikler profesyonel spor organizatörleri tarafindan yaygın olarak benimsenmiş ve verilerin kullanımı genel olarak profesyonel sporlarda sonuca etki eden potansiyel bir oyun değiştirici olarak kabul edilmektedir (Rein ve Memmert 2016). Benzer bir çalışmada ise Pappalardo ve arkadaşları (Pappalardo vd., 2019) bir müsabaka sırasında üretilen tüm olaylara ilişkin verileri depolayan ve analiz eden bir uygulama üretmişlerdir. PlayeRank adı verilen uygulamanın emsallerinden çok daha fazla veri toplayarak futbolcularla ilgili çok geniş istatistiki bilgiler vermesi yönüyle öne çıkmaktadır.

Fitness aktivitelerini izlemek ve değerlendirmek için "ideal" IoT tabanlı çözümü detaylandırmak amacıyla yapılan bir çalışmada (Akpa vd., 2019) analiz özellikli akıllı bir eldiven aracillğıyla fitness aktivitelerini izlemek için avuç içlerinin biyomekanik etkileşimlerinden yararlanma fikri sunulmaktadır. Akıllı eldiven tabanlı sistem, fitness seansları sırasında gözlemlenen avuç içlerindeki basınç dağılımının adet olarak sayılarını analiz ederek, fitness aktivitesini tanımlamak için kuvvete duyarlı direnç (FSR) sensörlerini giyilebilir fitness eldivenlerine entegre etmiştir. Sonuç olarak, el avuç içi basıncını toplayan ve analiz eden akıllı bir eldivenin fitness aktivitelerini izlemek ve değerlendirmek için kullanılabileceği sonucuna ulaşılmıştır. Bununla birlikte araştırmacılar; mevcut tasarımın, sistem avuç içlerinde baskı oluşturmayan egzersizleri izlemek için tek başına yeterli olmadığını bildirmektedir. Fitness uygulayıcılarını desteklemek için daha eksiksiz sistemler geliştirmek amacıyla atalet sensörlerinden gelen verilerle birleştirilerek daha iyi sonuçlara ulaşlabileceği aktarılmıştır.

Arttırılmıs Gerçeklik (AR) tabanlı laboratuvar testlerini alanda kullanmak amacıyla yapılan çalışmada bir voleybol sahasının görsel özelliklerini çoğaltarak algı-eylem ilişkilerini korurken, AR içeren bir sıçrama görevi ile voleybolculardaki diz yükünü ölçmek amaçlanmıştır. Bu çalışmanın bu nitelikteki ilk araştırma olduğu vurgulanmış ve gelecekteki araştırmaların AR'nin ortamlar arasında beceri aktarımını ve bu tür gelişim sağlayıcı sistemlerin geliştirme yeteneğini ve bunu yapmanın faydasını daha fazla araştırması zorunludur (Citraro vd., 2020).

Artırılmış Tırmanma Duvarı (ACW) tasarımının değerlendirmeye sunulduğu bir çalışmada (Kajastila, Holsti, ve Hamalainen 2016, ss.758-769) iç mekânda tırmanma deneyimi yaşamak isteyenleri motive etmek ve yönlendirmek için bilgisayarla görüntülü ve etkileşimli bir sistem tasarlanmıştır. Sistem hem çocuklar hem de yetişkinler de dâhil olmak üzere yüzlerce dağcı tarafindan rahatlıkla kullanılabilmektedir. ACW gelecekteki oyunların ve artırılmış sporların tasarlandığı yeni bir hareket tabanlı oyun sistemidir. Sistemle hareket ve zorlukların çeşitliliğini artırmak, başka türlü riskli bir ortamda kullanıcı tarafindan oluşturulan içeriği ve prosedürel olarak oluşturulmuş içeriği etkinleştirmek hedeflenmektedir. Artırılmış gerçeklik kullanarak gerçek boyutlu video tekrarına ve tırmanma rotası oluşturmaya olanak tanıyan benzer çalışmalar da (Wiehr vd., 2016) bulunmaktadır

\subsubsection{Sporda Şiddetin Önlenmesi ve Güvenlik}

Video izleme yoluyla şiddet tespit sistemi, halka açı yerlerde insanların güvenliğini artırmak için büyük ilgi görüyor. Ancak video gözetiminde insan tarafindan izlenen ve analiz edilen daha fazla sayıda kameranın kurulması gerekmektedir. Bu nedenle işlenecek veriler yoğundur ve şiddetin gerçek zamanlı olarak tespiti için optimal bir algoritma gerekmektedir. Bu nedenle, insan hatalarının oluşma olasıllğ1 ve sürekli izleme karmaşık bir iş haline gelir. Sorunun çözümüne ilişkin Fenil ve arkadaşaları tarafindan (Fenil vd., 2019, ss.191-192) sporcular ve seyirciler arasinda olası bir şiddetin meydana gelebileceği bir futbol stadyumu için şiddet tespit sistemi geliştirilmesi üzerine bir çalışma yapılmıştır. Şiddeti önceden önlemek için geliştirilen kamera odaklı sistem, eylemleri gerçek zamanlı olarak algılamakta, güvenlik güçlerini uyarmakta ve şiddeti \%94.5 oranında doğru tahmin ve tespit edebilmektedir. 
Ünlü, Y., \& Taş, Z. (2020). Sporda endüstri 4.0 uygulama alanları kapsamında yapılan araştırmalar üzerine bir bibliyografya denemesi. Journal of Human Sciences, 17(4), 1149-1176. doi:10.14687/jhs.v17i4.6088

\subsubsection{Spor Eğitimi ve Yetenek Gelişimi}

Bilgisayar teknolojisi, özellikle eğitim alanında giderek daha fazla kullanım alanı bulmaktadır. Öğretmenlere öğretimde yardımcı olmak, öğrencilerin öğrenmesine katk1 sağlamak, bilgisayar teknolojisini konulara dâhil etmek eğitim yöneticilerinin teknolojik gelişim sürecinde önemli bir görevi haline gelmiştir. Spor eğitim ve öğretiminde öğrencilere yardımc1 olacak bir simülasyon eğitim sistemi kurmak amacıyla bilgisayar sanal gerçeklik teknolojisini çekirdek olarak almak, bilgisayar teknolojisinin ilerlemesini ve genişletilebilirliğini daha iyi yansıtacağı düşünülmektedir (Haijun ve Yunli 2013).

Sporda görsel analiz olarak niteleyebileceğimiz bir çalışmada (Xiong vd., 2019, ss.103629103638) futbol antrenman videolarını analiz etmek için derin öğrenme tabanlı ayrıntılı eylem tanıma (FGAR) uygulaması geliştirilmiştir. Önerilen yöntem, bir oyuncunun futbol topunu başarıyla durdurup durdurmadığını değerlendirmek için kapalı alan antrenman ortamında ekipmanlar yardımıyla yapılan araştırmayı kapsamaktadır. İlk olarak, FGAR problemi insan-nesne (oyuncu-top) etkileşimleri olarak modellenmiştir. Nesne düzeyinde yörüngeler, ayrıntılı spor videolarını tanımlamak için yeni bir tanımlayıcı olarak önerilmektedir. Önerilen tanımlayıcı, üst düzey anlamsal ve insan-nesne etkileşim hareketlerinden yararlanabilmektedir. İkinci olarak, FGAR'1 gerçekleştirmek için nesne düzeyindeki yörüngelere dayalı derin ağların kademeli bir şeması önerilmektedir. Kademeli ağ, bir dedektör ağını bir sınıflandırıcı ağ (uzun-kısa-süreli bellek (LSTM) tabanlı bir ağ) ile birleştirerek oluşturmaktadır. Kademeli şema, dedektörün nesne algılama üzerindeki yüksek verimliliğinden ve işlem süresi serilerinde LSTM tabanlı ağın üstün performansından yararlanmaktadır. Bu araştırma önerilen yöntemin \% 93,24 doğruluk elde edebileceğini göstermektedir

Bu kapsamda yapılan çalışmalardan biri de IoT ve mobil internet tabanlı çeşitli spor aktivitelerinde iletişim sağlayan ve spora özgü deneyimlerin aktarılması temeline dayanan aynı zamanda bunların saptanması, düzenlenmesi ve değerlendirilmesi görevlerini yerine getiren bir uygulama tasarlanmıştır. Bu sayede sporcuların çeşitli spor aktivitelerinin tüm prosedürlerine (planlama, organizasyon, deneyim ve tartışma) katılmasına yardımcı olmak için çevrimiçi iletişim ve çevrimdış1 aktivite deneyimlerinin sık1 bir şekilde entegre edildiği bir O2O (Çevrimiçiden Çevrimdışına) spor topluluğu, internet plus arka planı altında önerilmektedir. Bu yeni spor topluluğu uygulamasında derin sosyal ilişki fikri ve yöntemleri ve büyük veri analizi uygulanmaktadır ( $\mathrm{Yu}$, 2018b, ss. 785-792). Araşturmacı benzer olarak bulut depolama tabanlı kişiselleştirilmiş spor etkinliği yönetimi uygulaması da tasarlamıştır (Yu, 2018a).

Yeni nesil raket sporları eğitimi için Nesnelerin İnterneti (IoT) tabanlı çalışmada (Wang vd., 2018, ss.4558-4566) performansın doğrulanması amaçlanmıştır. Bu çerçevede farklı badminton vuruşlarının tanınması ve farklı badminton oyuncularının beceri seviyelerinin sınıflandırılması için mikroelektromekanik sistemler hareket sensörlerine dayalı kablosuz bir giyilebilir algilama cihazı (WSD) kullanılmıştır. Sistem, veri toplama için özelleştirilmiş bir sensör düğümü, bir mobil uygulama ve bulut tabanlı bir veri işleme birimi içerir. Geliştirilen WSD, badminton vuruşlarını analiz etmek için video tabanlı yöntemlere kıyasla düşük maliyetli, kullanımı kolay ve hesaplama açısından verimli bir uygulamadır. Sistem aynı zamanda iç ve dış ortamlarda birden fazla oyuncunun dinamik izleme avantajını sunmaktadır.

Raket sporlarına yönelik hareket parametrelerini etkili bir şekilde algılamak amacıyla yapılan bir çalışmada (Xia vd., 2020, ss.1638) güvenilir aktivite tanımlama ve sporcu hareketlerinin sayımını gerçekleştirmek amacıyla bir raket sporu tanıma bilekliği sistemi ve çok katmanlı bir hibrit kümeleme modeli geliştirilmiştir. Sistem bir bluetooth örgü ağı, bir telefon ve bileklik arasında iletişim sağlamakta ve birden çok cihaz arasında bağlantı kurmaktadır. Bu, kullanıcıların egzersizlerini telefon aracıllğıyla takip etmelerine ve diğer oyuncular ve hakemlerle bilgi paylaşmalarına olanak tanmaktadır. Böylece sporcuların hareketi doğru olarak gözlemleyip kendinden beklenen hareketi doğru olarak yapmalarına ve hatalı olan hareketlerin de anında çevreye bildirilmesini sağlamaktadır.

IoT teknolojisi ile toplanan verilerin analizine dayanan bir çalışmada (Liu vd., 2020, ss.3123431244) kürek sporcularının durumunu sürekli izleyen ve yetenekli kürekçilerin seçiminde anahtar rol oynadığı görülen bir sistem geliştirilmiştir. Bu sisteme göre kürek sporcularının seçiminde kürekçilerin fiziksel özelliklerinin en önemli gösterge olduğu belirlenmiş, uygun vücut tipinin avantajına sahip 
olmanın, gelecekte antrenmanı daha etkili hale getirebileceği bildirilmiştir. Araştırma sonuçlanı, "vücut faktörü" kriterinin en önemli seçim göstergesi olduğunu ve ardından "IoT" kriterinin geldiğini göstermektedir. Doğal olarak uygun vücut tipine sahip bir sporcunun, diğer vücut tiplerine sahip sporcular tarafindan elde edilemeyen bir avantajının olduğu ifade edilmektedir. Çalışmada bu vücut tipindeki sporcuları ayırmak için Büyük Veri analizi yoluyla ve Io'T teknolojisinin kullanımıyla izleme ve seçim sürecinin daha objektif önlemler sağlayabileceği aktarılmıştır.

Zihinsel engelli bireylerin yüzme performansına yönelik yapılan analizlerde de atalet sensörü ve kamera kullanılarak yapılan çalışmalar (Caporaso vd., 2020) yüzme eğitimini izlemek ve iyileştirmek adına alana önemli katkılar sağlamaktadır.

Baudisch ve arkadaşları (Baudisch vd., 2014, ss.575-578) gerçek ve görünür bir basketbol topu olmadan, taklite dayanan sanal gerçeklik tabanlı bir uygulama geliştirmiştir. Çalışmada katulımcılara temel bir basketbol eğitimi verilmesi amaçlanmıştır. Çalışmada katulımcılara az miktarda işitsel geri bildirimler vermektedir. Çalışma aynı zamanda sporcuların birbirlerinin hareketlerini izleyerek pozisyon almalarına böylece öğrenme süreçlerine imkân tanıyan görünmez top kavramı etrafindaki yeni bir oyun dinamiğine dayanmaktadır.

\subsection{Sporda Endüstri 4.0 Uygulama Alanları Kapsamında Yapılan Araştırmalaralar 2.4.1.Giyilebilir ak1llı cihazlar}

1. Ahsan, M., et al. (2020). "Development of Novel Big Data Analytics Framework for Smart Clothing." Ieee Access 8: 146376-146394.

2. Akpa, A., et al. (2019). "A smart glove to track fitness exercises by reading hand palm." 2019.

3. Barshan, B. and A. Yurtman (2020). "Classifying Daily and Sports Activities Invariantly to the Positioning of Wearable Motion Sensor Units." Ieee Internet of Things Journal 7(6): 4801-4815.

4. Dargazany, A. R., et al. (2018). "WearableDL: Wearable Internet-of-Things and Deep Learning for Big Data Analytics-Concept, Literature, and Future." Mobile Information Systems 2018.

5. Dei, M., et al. (2019). "CMOS Interfaces for Internet-of-Wearables Electrochemical Sensors: Trends and Challenges." Electronics 8(2).

6. Delabrida, S., et al. (2016). "Wearable HUD for Ecological Field Research Applications." Mobile Networks \& Applications 21(4): 677-687.

7. Dian, F. J., et al. (2020). "Wearables and the Internet of Things (IoT), Applications, Opportunities, and Challenges: A Survey." Ieee Access 8: 69200-69211.

8. Duking, P., et al. (2018). "Integrated Framework of Load Monitoring by a Combination of Smartphone Applications, Wearables and Point-of-Care Testing Provides Feedback that Allows Individual Responsive Adjustments to Activities of Daily Living." Sensors 18(5).

9. Ebling, M. R. (2016). "IoT: From Sports to Fashion and Everything In-Between." Ieee Pervasive Computing 15(4): 2-4.

10. Ferri, J., et al. (2017). "A Wearable Textile 2D Touchpad Sensor Based on Screen-Printing Technology." Materials (Basel) 10(12).

11. Ferri, J., et al. (2018). "Integration of a 2D Touch Sensor with an Electroluminescent Display by Using a Screen-Printing Technology on Textile Substrate." Sensors (Basel) 18(10).

12. Guan, H. Y., et al. (2019). "A self-powered wearable sweat-evaporation-biosensing analyzer for building sports big data." Nano Energy 59: 754-761

13. Hamalainen, M., et al. (2020). "ETSI SmartBAN Architecture: The Global Vision for Smart Body Area Networks." Ieee Access 8: 150611-150625. 

denemesi. Journal of Human Sciences, 17(4), 1149-1176. doi:10.14687/jhs.v17i4.6088

14. Huynh, N. T. and C. F. Chien (2018). "A hybrid multi-subpopulation genetic algorithm for textile batch dyeing scheduling and an empirical study." Computers \& Industrial Engineering 125: 615-627.

15. James, D. A. and N. Petrone (2016). Sensors and Wearable Technologies in Sport: Technologies, Trends and Approaches for Implementation, Springer.

16. Kao, C. H., et al. (2018). "Novel digital glove design for virtual reality applications." Microsystem Technologies-Micro-and Nanosystems-Information Storage and Processing Systems 24(10): 4247-4266.

17. Kos, A. and A. Umek (2019). "Wearable Sensor Devices for Prevention and Rehabilitation in Healthcare: Swimming Exercise With Real-Time Therapist Feedback." Ieee Internet of Things Journal 6(2): 1331-1341.

18. Krishnan, S., et al. (2018). "Internet of things for knowledge administrations by wearable gadgets." Journal of Medical Systems 42(11).

19. Li, Y. J., et al. (2019). "Touch switch sensor for cognitive body sensor networks." Computer Communications 146: 32-38.

20. Liu, B., et al. (2020). "Self-powered Biosensor Big Data Intelligent Information Processing System for Real-time Motion Monitoring." Zeitschrift Fur Anorganische Und Allgemeine Chemie 646(11-12): 500-506.

21. Marin, J., et al. (2017). "Octopus: A Design Methodology for Motion Capture Wearables." Sensors 17(8).

22. Ravi, D., et al. (2017). "A Deep Learning Approach to on-Node Sensor Data Analytics for Mobile or Wearable Devices." Ieee Journal of Biomedical and Health Informatics 21(1): 5664.

23. Resta, P., et al. (2020). A wearable system for knee flexion/extension monitoring: design and assessment. 2020 IEEE International Workshop on Metrology for Industry 4.0 \& IoT, IEEE.

24. Segura-Garcia, J., et al. (2018). "Cost-Effective eHealth System Based on a Multi-Sensor System-on-Chip Platform and Data Fusion in Cloud for Sport Activity Monitoring." Electronics 7(9).

25. Huque, M. T. I., et al. (2015). "Body Node Coordinator Placement Algorithms for Wireless Body Area Networks." Ieee Internet of Things Journal 2(1): 94-102.

26. Xia, K., et al. (2020). "Racquet Sports Recognition Using a Hybrid Clustering Model Learned from Integrated Wearable Sensor." 20(6): 1638.

27. Zhang, W. L. H., et al. (2020). "Wearable Battery-Free Perspiration Analyzing Sites Based on Sweat Flowing on ZnO Nanoarrays." Nano-Micro Letters 12(1).

28. Zhao, J. Y. and G. X. Li (2020). "Study on real-time wearable sport health device based on body sensor networks." Computer Communications 154: 40-47.

\subsubsection{Performans ve Analiz}

1. Andrienko, G., et al. (2017). "Visual analysis of pressure in football." Data Mining and Knowledge Discovery 31(6): 1793-1839.

2. Assuncao, R. and K. Pelechrinis (2018). "Sports Analytics in the Era of Big Data: Moving Toward the Next Frontier." Big Data 6(4): 237-238.

3. Avila-Moreno, F. M., et al. (2018). "Evaluation of tactical performance in invasion team sports: a systematic review." International Journal of Performance Analysis in Sport 18(2): 195-216.

4. Beal, R., et al. (2019). "Artificial intelligence for team sports: a survey." Knowledge Engineering Review 34. 
Ünlü, Y., \& Taş, Z. (2020). Sporda endüstri 4.0 uygulama alanları kapsamında yapılan araştırmalar üzerine bir bibliyografya denemesi. Journal of Human Sciences, 17(4), 1149-1176. doi:10.14687/jhs.v17i4.6088

5. Belem, I., et al. (2016). "Stress in Mma: Can Coping Strategies Improve the Performance of Fighters?" Revista Brasileira De Medicina Do Esporte 22(4): 287-290.

6. Bonidia, R. P., et al. (2018). "Data Mining in Sports: A Systematic Review." Ieee Latin America Transactions 16(1): 232-239.

7. Burk, V., et al. (2016). "'Same, Same-but Different!" On Consumers' Use of Corporate PR Media in Sports." Journal of Sport Management 30(4): 353-368.

8. Caporaso, T., et al. (2020). A preliminary approach for swimming performance analysis of fisdir elite athletes with intellectual impairment using an inertial sensor. 2020 IEEE International Workshop on Metrology for Industry 4.0 \& IoT, IEEE.

9. Carpita, M., et al. (2019). "Exploring and modelling team performances of the Kaggle European Soccer database." Statistical Modelling 19(1): 74-101.

10. Catalani, V., et al. (2018). "Octodrine: New Questions and Challenges in Sport Supplements." Brain Sciences 8(2).

11. Chang, P. D., et al. (2019). "Deep Learning for Detection of Complete Anterior Cruciate Ligament Tear." Journal of Digital Imaging 32(6): 980-986.

12. Chittenden, T. (2017). "Skin in the game: the use of sensing smart fabrics in tennis costume as a means of analyzing performance." Fashion and Textiles 4.

13. Chu, W. C. C., et al. (2019). "Artificial Intelligence of Things in Sports Science: Weight Training as an Example." Computer 52(11): 52-61.

14. Chung, D. J. (2017). "How Much Is a Win Worth? An Application to Intercollegiate Athletics." Management Science 63(2): 548-565.

15. Cronin, N. J., et al. (2019). "Markerless 2D kinematic analysis of underwater running: A deep learning approach." Journal of Biomechanics 87: 75-82.

16. Cusano, P., et al. (2019). "New Technologies in Sports to Improve Biomechanical Aspects, Performance and Training Evaluation." Acta Medica Mediterranea 35(6): 3009-3012.

17. De Oliveira, F. A., et al. (2019). "Implementation and Performance of the Gas Chromatography/Combustion/Isotope Ratio Mass Spectrometry-Based Method for the Confirmatory Analysis of Endogenous Anabolic Steroids during the Rio de Janeiro Olympic and Paralympic Games 2016." Analytical Chemistry 91(18): 11747-11756.

18. Ding, P. (2019). "The revolution of sports communication in the age of big data." Basic \& Clinical Pharmacology \& Toxicology 125: 226-227.

19. Doyle, J. R. and P. A. Bottomley (2019). "The relative age effect in European elite soccer: A practical guide to Poisson regression modelling." PLoS One 14(4).

20. Dunstan, R. H., et al. (2020). "Modelling of amino acid turnover in the horse during training and racing: A basis for developing a novel supplementation strategy." PLoS One 15(1): e0226988.

21. Elumalai, G. and R. Ramakrishnan (2020). "A Novel Approach to Monitor and Maintain Database About Physiological Parameters of (Javelin) Athletes Using Internet of Things (IoT)." Wireless Personal Communications 111(1): 343-355.

22. Elumalai, G. and R. Ramakrishnan (2020). "Development of novel and efficient approach for analyzing and monitoring the movement parameters for javelin athletes based on internet of things." Journal of Ambient Intelligence and Humanized Computing.

23. Erickson, K., et al. (2016). "'The ripples are big": Storying the impact of doping in sport beyond the sanctioned athlete." Psychology of Sport and Exercise 24: 92-99.

24. Fitzgerald, C. F. and R. L. Jensen (2020). "A Comparison of the National Football League's Annual National Football League Combine 1999-2000 to 2015-2016." Journal of Strength and Conditioning Research 34(3): 771-781. 

denemesi. Journal of Human Sciences, 17(4), 1149-1176. doi:10.14687/jhs.v17i4.6088

25. Flatmo, F., et al. (2019). "Musculoskeletal complaints among professional divers." Int Marit Health 70(2): 107-112.

26. Geeroms, J., et al. (2017). "Design and energetic evaluation of a prosthetic knee joint actuator with a lockable parallel spring." Bioinspir Biomim 12(2): 026002.

27. Goes, F. R., et al. (2020). "Unlocking the potential of big data to support tactical performance analysis in professional soccer: A systematic review." European Journal of Sport Science.

28. Gonzalez-Gonzalez, C. S., et al. (2018). "Exploring the Benefits of Using Gamification and Videogames for Physical Exercise: a Review of State of Art." International Journal of Interactive Multimedia and Artificial Intelligence 5(2): 46-52.

29. Greenbaum, D. (2018). "Wuz You Robbed? Concerns With Using Big Data Analytics in Sports." American Journal of Bioethics 18(6): 32-33.

30. Grooms, D. R., et al. (2018). "Brain-Behavior Mechanisms for the Transfer of Neuromuscular Training Adaptions to Simulated Sport: Initial Findings From the Train the Brain Project." Journal of Sport Rehabilitation 27(5).

31. Hammad, I. and K. El-Sankary (2019). "Practical Considerations for Accuracy Evaluation in Sensor-Based Machine Learning and Deep Learning." Sensors 19(16

32. Hoeber, O., et al. (2016). "Visual Twitter Analytics (Vista) Temporally changing sentiment and the discovery of emergent themes within sport event tweets." Online Information Review 40(1): 25-41.

33. Hulsmann, F., et al. (2018). "Classification of motor errors to provide real-time feedback for sports coaching in virtual reality - A case study in squats and Tai Chi pushes." Computers \& Graphics-Uk 76: 47-59.

34. Hung, T. H., et al. (2014). "Examining the relationship between cardiorespiratory fitness and body weight status: empirical evidence from a population-based survey of adults in Taiwan." ScientificWorldJournal.

35. Janssen, M., et al. (2020). "Understanding Different Types of Recreational Runners and How They Use Running-Related Technology." International Journal of Environmental Research and Public Health 17(7).

36. Jia, L. (2017). "Professional basketball club training management based on the big data thinking method." Agro Food Industry Hi-Tech 28(1): 2012-2017.

37. Jiang, R. and Y. P. Li (2020). "Dynamic pricing analysis of redundant time of sports culture hall based on big data platform." Personal and Ubiquitous Computing 24(1): 19-31.

38. Kajastila, R., et al. (2016). "The Augmented Climbing Wall: High-Exertion Proximity Interaction on a Wall-Sized Interactive Surface." 34th Annual Chi Conference on Human Factors in Computing Systems, Chi 2016: 758-769.

39. Kamkar, S., et al. (2020). "Multiple-target tracking in human and machine vision." Plos Computational Biology 16(4).

40. Kazemipoor, M., et al. (2020). "Computational Intelligence Techniques for Assessing Anthropometric Indices Changes in Female Athletes." Current Medical Imaging 16(4): 288295.

41. Khan, M. A., et al. (2020). "Human action recognition using fusion of multiview and deep features: an application to video surveillance." Multimedia Tools and Applications.

42. Kim, H., et al. (2018). "Replacing Self-Efficacy in Physical Activity: Unconscious Intervention of the AR Game, Pokemon GO." Sustainability 10(6).

43. Kim, J., et al. (2018). "Athlete personality characteristics and informal role occupancy in interdependent sport teams." Psychology of Sport and Exercise 39: 193-203. 

denemesi. Journal of Human Sciences, 17(4), 1149-1176. doi:10.14687/jhs.v17i4.6088

44. Kim, S., et al. (2020). "Implementation of user playstyle coaching using video processing and statistical methods in league of legends." Multimedia Tools and Applications.

45. Kotnik, K. Z., et al. (2018). "Use of dietary supplements in differently physically active adolescents." Journal of Food and Nutrition Research 57(3): 231-241.

46. Kremmer, C. (2017). "Racing chemistry: A century of challenges and progress." Drug Test Anal 9(9): 1284-1290.

47. Lapadula, V., et al. (2020). A Body Hydration Analysis System to improve running performance. 2020 IEEE International Workshop on Metrology for Industry 4.0 \& IoT, IEEE.

48. Le Noury, P., et al. (2020). "Examining the representativeness of a virtual reality environment for simulation of tennis performance." Journal of Sports Sciences.

49. Lee, Y. S., et al. (2020). "Data Mining Analysis of Overall Team Information Based on Internet of Things." Ieee Access 8: 41822-41829.

50. Leutzinger, T. J., et al. (2018). "Anthropometric and Athletic Performance Combine Test Results among Positions within Grade Levels of High School-Aged American Football Players." Journal of Strength and Conditioning Research 32(5): 1288-1296.

51. Li, D. M., et al. (2020). "Research on the inheritance and protection of data mining technology in national sports." Concurrency and Computation-Practice \& Experience.

52. Li, G. J. and C. P. Zhang (2019). "Research on static image recognition of sports based on machine learning." Journal of Intelligent \& Fuzzy Systems 37(5): 6205-6215.

53. Liang, C. M. (2019). "Prediction and analysis of sphere motion trajectory based on deep learning algorithm optimization." Journal of Intelligent \& Fuzzy Systems 37(5): 6275-6285.

54. Lida, H. and M. N. A. Khalid (2020). "Using Games to Study Law of Motions in Mind." Ieee Access 8: 138701-138709.

55. Link, D. (2016). "Symposium: BIG Data in Sport Games." Research Quarterly for Exercise and Sport 87: S58-S58.

56. Liu, F. C., et al. (2020). "An Intelligent Multi-View Active Learning Method Based on a Double-Branch Network." Entropy 22(8).

57. Lucrezi, S., et al. (2018). "Stirring the strategic direction of scuba diving marine Citizen Science: A survey of active and potential participants." PLoS One 13(8): e0202484.

58. Ma, H. M. and X. F. Pang (2019). "Research and Analysis of Sport Medical Data Processing Algorithms Based on Deep Learning and Internet of Things." Ieee Access 7: 118839-118849.

59. Ma, H. M. and X. F. Pang (2019). "Research and Analysis of Sport Medical Data Processing Algorithms Based on Deep Learning and Internet of Things." Ieee Access 7: 118839-118849.

60. Mahmood, Z., et al. (2015). "Automatic player detection and identification for sports entertainment applications." Pattern Analysis and Applications 18(4): 971-982.

61. Mahmoudi, S. A., et al. (2020). "Multimedia processing using deep learning technologies, high-performance computing cloud resources, and Big Data volumes." Concurrency and Computation-Practice \& Experience 32(17).

62. Marszalek, J. and B. Molik (2019). "Reliability of measurement of active trunk movement in wheelchair basketball players." PLoS One 14(11).

63. Massaroni, C., et al. (2020). Respiratory monitoring during cycling exercise: performance assessment of a smart t-shirt embedding fiber optic sensors. 2020 IEEE International Workshop on Metrology for Industry 4.0 \& IoT, IEEE.

64. McHale, I. G. and S. D. Relton (2018). "Identifying key players in soccer teams using network analysis and pass difficulty." European Journal of Operational Research 268(1): 339-347. 

denemesi. Journal of Human Sciences, 17(4), 1149-1176. doi:10.14687/jhs.v17i4.6088

65. McLean, B. D., et al. (2019). "Quantifying Physical Demands in the National Basketball Association-Challenges Around Developing Best-Practice Models for Athlete Care and Performance." International Journal of Sports Physiology and Performance 14(4): 414-420.

66. Mecheri, S., et al. (2016). "The serve impact in tennis: First large-scale study of big Hawk-Eye data." Statistical Analysis and Data Mining 9(5): 310-325.

67. Musa, R. M., et al. (2019). "The application of Artificial Neural Network and k-Nearest Neighbour classification models in the scouting of high-performance archers from a selected fitness and motor skill performance parameters." Science \& Sports 34(4): E241-E249.

68. Pappalardo, L., et al. (2019). "PlayeRank: Data-driven Performance Evaluation and Player Ranking in Soccer via a Machine Learning Approach." Acm Transactions on Intelligent Systems and Technology 10(5).

69. Park, E. M., et al. (2016). "The effects of leadership by types of soccer instruction on big data analysis." Cluster Computing-the Journal of Networks Software Tools and Applications 19(3): 1647-1658

70. Park, S. U., et al. (2020). "Big Data Analysis of Sports and Physical Activities among Korean Adolescents." International Journal of Environmental Research and Public Health 17(15).

71. Rein, R. and D. Memmert (2016). Big data and tactical analysis in elite soccer. Future challenges and opportunities for sports science. SpringerPlus 5 (1): 1410.

72. Rodrigues, A. C. N., et al. (2020). "Using Artificial Intelligence for Pattern Recognition in a Sports Context." Sensors 20(11).

73. Santuz, A., et al. (2018). "Modular Control of Human Movement During Running: An Open Access Data Set." Frontiers in Physiology 9.

74. Song, W., et al. (2019). "Design and Implementation of Beach Sports Big Data Analysis System Based on Computer Technology." Journal of Coastal Research: 327-331.

75. Sun, L. W. (2019). "Research on classification and recognition of badminton batting action based on machine learning." Journal of Intelligent \& Fuzzy Systems 37(5): 6241-6252.

76. Urbaczewski, A. and R. Elmore (2018). "Big Data, Efficient Markets, and the End of Daily Fantasy Sports As We Know It?" Big Data 6(4): 239-247.

77. Vinue, G. (2020). "A Web Application for Interactive Visualization of European Basketball Data." Big Data 8(1): 70-86.

78. Visscher, R. M. S., et al. (2019). "Artificial intelligence for understanding concussion: Retrospective cluster analysis on the balance and vestibular diagnostic data of concussion patients." PLoS One 14(4).

79. Wang, I. L., et al. (2019). "The effects of Jilin sika Deer's (Cervus dybowski) tendon liquid supplementation on endurance drop jumps performance, biochemistry profile of free boxing players." J Ethnopharmacol 245: 112119.

80. Wang, J. and B. Lv (2019). "Big data analysis and research on consumption demand of sports fitness leisure activities." Cluster Computing-the Journal of Networks Software Tools and Applications 22(2): S3573-S3582.

81. Wiehr, F., et al. (2016). betaCube: Enhancing training for climbing by a self-calibrating camera-projection unit. Proceedings of the 2016 CHI Conference Extended Abstracts on Human Factors in Computing Systems.

82. Xue, B. H. and T. Liu (2019). "Research on emotional model of sports arena based on artificial intelligence emotion calculation." Cluster Computing-the Journal of Networks Software Tools and Applications 22: 14927-14933.

83. Yi, Q., et al. (2018). "Technical demands of different playing positions in the UEFA Champions League." International Journal of Performance Analysis in Sport 18(6): 926-937. 

denemesi. Journal of Human Sciences, 17(4), 1149-1176. doi:10.14687/jhs.v17i4.6088

84. Yu, L. (2018). "Cloud storage-based personalized sports activity management in Internet plus O2O sports community." Concurrency and Computation-Practice \& Experience 30(24).

85. Yu, L. J. C. S. R. (2018). "Sports activity detection, organization and evaluation in online to offline sports community." 52: 785-792.

86. Yu, L. J. C., et al. (2018). "Cloud storage-based personalized sports activity management in Internet plus $\mathrm{O} 2 \mathrm{O}$ sports community." 30(24): e4932.

87. Zambom-Ferraresi, F., et al. (2018). "Determinants of sport performance in European football: What can we learn from the data?" Decision Support Systems 114: 18-28.

88. Zhan, Y. Z. and K. H. Tan (2020). "An analytic infrastructure for harvesting big data to enhance supply chain performance." European Journal of Operational Research 281(3): 55957

89. Zhang, B. and D. X. Chen (2020). "Resource scheduling of green communication network for large sports events based on edge computing." Computer Communications 159: 299-309.

90. Zhang, J. Y., et al. (2019). "Analysis Method of motion Information Driven by Medical Big Data." Ieee Access 7: 174189-174199.

91. Zhang, K. L., et al. (2020). "An automatic multi-camera-based event extraction system for real soccer videos." Pattern Analysis and Applications 23(2): 953-965.

92. Zhang, L. (2020). "Design of a sports culture data fusion system based on a data mining algorithm." Personal and Ubiquitous Computing 24(1): 75-86.

93. Zhang, X. R. and J. H. Sun (2019). "Discussion on new media communication strategy of sports events based on large data technology." Cluster Computing-the Journal of Networks Software Tools and Applications 22(2): S3395-S3403.

\subsubsection{Tahmin tabanlı uygulama ve programlar}

1. Abraham, C., et al. (2015). "Photonic non-contact estimation of blood lactate level." Biomed Opt Express 6(10): 4144-4153.

2. Baughman, A. K., et al. (2015). "Predictive Cloud Computing with Big Data: Professional Golf and Tennis Forecasting." Ieee Computational Intelligence Magazine 10(3): 62-76.

3. Baughman, A. K., et al. (2016). "IBM Predicts Cloud Computing Demand for Sports Tournaments." Interfaces 46(1): 33-48.

4. Behravan, I., et al. (2019). "Finding Roles of Players in Football Using Automatic Particle Swarm Optimization-Clustering Algorithm." Big Data 7(1): 35-56.

5. Bialkowski, A., et al. (2016). "Discovering Team Structures in Soccer from Spatiotemporal Data." Ieee Transactions on Knowledge and Data Engineering 28(10): 2596-2605.

6. Collins, D. and L. Collins (2020). "Developing coaches' professional judgement and decision making: Using the 'Big 5'." Journal of Sports Sciences.

7. Fialho, G., et al. (2019). "Predicting Sports Results with Artificial Intelligence-A Proposal Framework for Soccer Games." 164: 131-136.

8. Gaudino, P., et al. (2014). "Estimated metabolic and mechanical demands during different small-sided games in elite soccer players." Human Movement Science 36: 123-133.

9. Gu, W., et al. (2019). "A game-predicting expert system using big data and machine learning." Expert Systems with Applications 130: 293-305.

10. Hancock, L., et al. (2018). "Applying Corporate Political Activity (CPA) analysis to Australian gambling industry submissions against regulation of television sports betting advertising." PLoS One 13(10)

11. Hargrove, J. S., et al. (2015). "Using Tournament Angler Data to Rapidly Assess the Invasion Status of Alien Sport Fishes (Micropterus spp.) in Southern Africa." PLoS One 10(6). 

denemesi. Journal of Human Sciences, 17(4), 1149-1176. doi:10.14687/jhs.v17i4.6088

12. Hasan, W. B., et al. (2019). "Adaptive User Grouping Based on EVM Prediction for Efficient Robust Massive MIMO in TDD." Ieee Access 7: 162683-162696.

13. Hassan, A., et al. (2020). "Predicting Wins, Losses and Attributes' Sensitivities in the Soccer World Cup 2018 Using Neural Network Analysis." Sensors 20(11).

14. Hsu, Y. C. (2020). "Using Machine Learning and Candlestick Patterns to Predict the Outcomes of American Football Games." Applied Sciences-Basel 10(13).

15. Itoh, Y., et al. (2016). Laplacian vision: Augmenting motion prediction via optical see-through head-mounted displays. Proceedings of the 7th Augmented Human International Conference 2016.

16. Keshtkar Langaroudi, M., et al. (2019). "Sports result prediction based on machine learning and computational intelligence approaches: A survey." 5(1): 27-36.

17. Lee, J. Y. and T. Woo (2018). "A novel method for natural motion mapping as a strategy of game immediacy." Ksii Transactions on Internet and Information Systems 12(5): 2313-2326.

18. Li, D. M., et al. (2020). "Research on the inheritance and protection of data mining technology in national sports." Concurrency and Computation-Practice \& Experience.

19. Li, T. J., et al. (2019). "Competition prediction and fitness behavior based on GA-SVM algorithm and PCA model." Journal of Intelligent \& Fuzzy Systems 37(5): 6191-6203.

20. Muller, O., et al. (2017). "Beyond crowd judgments: Data-driven estimation of market value in association football." European Journal of Operational Research 263(2): 611-624.

21. Oytun, M., et al. (2020). "Performance Prediction and Evaluation in Female Handball Players Using Machine Learning Models." Ieee Access 8: 116321-116335.

22. Rotshtein, A. P., et al. (2005). "Football predictions based on a fuzzy model with genetic and neural tuning." 41(4): 619-630.

23. Seo, S. and D. Kang (2016). "Study on predicting sentiment from images using categorical and sentimental keyword-based image retrieval." Journal of Supercomputing 72(9): 34783488.

24. Sim, M. K. and D. G. Choi (2020). "The Winning Probability of a Game and the Importance of Points in Tennis Matches." Research Quarterly for Exercise and Sport 91(3): 361-372.

25. Staartjes, V. E., et al. (2019). "Deep learning-based preoperative predictive analytics for patient-reported outcomes following lumbar discectomy: feasibility of center-specific modeling." Spine Journal 19(5): 853-861.

26. Thompson, M. P., et al. (2019). "Risk Management and Analytics in Wildfire Response." Current Forestry Reports 5(4): 226-239.

27. Yang, K. (2020). "The construction of sports culture industry growth forecast model based on big data." Personal and Ubiquitous Computing 24(1): 5-17.

28. Yeh, Y. E. (2020). "Prediction of Optimized Color Design for Sports Shoes Using an Artificial Neural Network and Genetic Algorithm." Applied Sciences-Basel 10(5).

\subsubsection{Spor Malzeme ve Materyalleri}

1. Ali, U., et al. (2018). "Conceptualization, Design and Development of the Football Bladder Making Machine under the concept of Industry 4.0." 13(9): 218-222.

2. Avramescu, R. E., et al. (2018). "Superhydrophobic Natural and Artificial Surfaces-A Structural Approach." Materials (Basel) 11(5).

3. Bacic, B. (2016). "Predicting golf ball trajectories from swing plane: An artificial neural networks approach." Expert Systems with Applications 65: 423-438.

4. Bermudez, G. S. C., et al. (2018). "Magnetosensitive e-skins with directional perception for augmented reality." Science Advances 4(1). 

denemesi. Journal of Human Sciences, 17(4), 1149-1176. doi:10.14687/jhs.v17i4.6088

5. Bernardina, G. R. D., et al. (2019). "Moving system with action sport cameras: 3D kinematics of the walking and running in a large volume." PLoS One 14(11).

6. Casas, O. V., et al. (2016). "3D load cell for measure force in a bicycle crank." Measurement 93: 189-201.

7. Chen, X. J., et al. (2017). "A cloud computing architecture for characterization and classification of moving object." Multimedia Tools and Applications 76(16): 17319-17336.

8. Duncan, O., et al. (2018). "Review of auxetic materials for sports applications: Expanding options in comfort and protection." 8(6): 941.

9. Edwards, J. (2020). "Photo and Video Technologies Target New Frontiers: Innovative Imaging Research Enabled by Signal Processing Is Making Cameras More Powerful and Versatile [Special Reports]." Ieee Signal Processing Magazine 37(3): 8

10. Ewurum, C. H., et al. (2018). "Surgical Navigation in Orthopedics: Workflow and System Review." Intelligent Orthopaedics: Artificial Intelligence and Smart Image-Guided Technology for Orthopaedics 1093: 47-63.

11. Haegele, J. A., et al. (2019). "Paraeducator Support in Integrated Physical Education as Reflected by Adults With Visual Impairments." Adapted Physical Activity Quarterly 36(1): 91-108.

12. Huang, H. L., et al. (2020). "Multiple Stimuli Responsive and Identifiable Zwitterionic Ionic Conductive Hydrogel for Bionic Electronic Skin." Advanced Electronic Materials 6(7).

13. Jetzschke, S., et al. (2017). "Finding Home: Landmark Ambiguity in Human Navigation." Frontiers in Behavioral Neuroscience 11.

14. Knittel, D., et al. (2020). "Milling diagnosis using artificial intelligence approaches." Mechanics \& Industry 20:8

15. Kwag, S., et al. (2020). "Optimal seat allocation strategy for e-sports gaming center." International Transactions in Operational Research.

16. Laverdiere, C., Et Al. (2019). "Augmented Reality İn Orthopaedics A Systematıc Review And A Window On Future Possibilities." Bone \& Joint Journal 101b(12): 1479-1488.

17. Li, R. (2020). "Evaluation and simulation of medical sports health equipment multimedia image based on information asymmetry theory." Multimedia Tools and Applications 79(1516): 9957-9976.

18. Liu, H. B., et al. (2020). "Flexible and Degradable Multimodal Sensor Fabricated by Transferring Laser-Induced Porous Carbon on Starch Film." Acs Sustainable Chemistry \& Engineering 8(1): 527-533.

19. Lu, Y. and S. Y. An (2020). "Research on sports video detection technology motion 3D reconstruction based on hidden Markov model." Cluster Computing-the Journal of Networks Software Tools and Applicatio

20. Luo, J. J., et al. (2019). "Flexible and durable wood-based triboelectric nanogenerators for self-powered sensing in athletic big data analytics." Nature Communications 10

21. Mahmood, Z., et al. (2017). "EAR: Enhanced Augmented Reality System for Sports Entertainment Applications." Ksii Transactions on Internet and Information Systems 11(12): 6069-6091.

22. Merriaux, P., et al. (2017). "A Study of Vicon System Positioning Performance." Sensors 17(7).

23. Moon, J., et al. (2015). "Effect of ski simulator training on kinematic and muscle activation of the lower extremities." Journal of Physical Therapy Science 27(8): 2629-2632.

24. Mora, H., et al. (2017). "An IoT-Based Computational Framework for Healthcare Monitoring in Mobile Environments." Sensors 17(10). 
Ünlü, Y., \& Taş, Z. (2020). Sporda endüstri 4.0 uygulama alanları kapsamında yapılan araştırmalar üzerine bir bibliyografya denemesi. Journal of Human Sciences, 17(4), 1149-1176. doi:10.14687/jhs.v17i4.6088

25. Qi, W., et al. (2019). "A Fast and Robust Deep Convolutional Neural Networks for Complex Human Activity Recognition Using Smartphone." Sensors 19(17).

26. Ranjan, R. and W. S. Gan (2015). "Natural Listening over Headphones in Augmented Reality Using Adaptive Filtering Techniques." Ieee-Acm Transactions on Audio Speech and Language Processing 23(11): 1988-2002.

27. Rigamonti, L., et al. (2020). "Potentials of Digitalization in Sports Medicine: A Narrative Review." Current Sports Medicine Reports 19(4): 157-163.

28. Sainio, J., et al. (2015). "Generating Heat Maps of Popular Routes Online from Massive Mobile Sports Tracking Application Data in Milliseconds While Respecting Privacy." Isprs International Journal of Geo-Information 4(4): 1813-1826.

29. Salisbury, J. P., et al. (2018). "Concussion Assessment With Smartglasses: Validation Study of Balance Measurement Toward a Lightweight, Multimodal, Field-Ready Platform." Jmir Mhealth and Uhealth 6(1).

30. Silva, F. M. P., et al. (2019). "Comparative study of the manufacturing technologies of Sports Bras aiming at adjusting the productive processes of the Brazilian apparel enterprises to the model Industry 4.0." Textiles, Identity and Innovation: Design the Future: 125-130.

31. Sun, J., et al. (2020). "Mapping Essential Urban Land Use Categories in Nanjing by Integrating Multi-Source Big Data." Remote Sensing 12(15).

32. Van der Slikke, R. M. A., et al. (2016). "From big data to rich data: The key features of athlete wheelchair mobility performance." Journal of Biomechanics 49(14): 3340-3346V

33. Van der Slikke, R. M. A., et al. (2018). "The Future of Classification in Wheelchair Sports: Can Data Science and Technological Advancement Offer an Alternative Point of View?" International Journal of Sports Physiology and Performance 13(6): 742-749.

34. Vines, J. B., et al. (2018). "Contemporary Polymer-Based Nanoparticle Systems for Photothermal Therapy." Polymers (Basel) 10(12).

35. Weber, A. (2018). "Smart Manufacturing Stakeholders and Their Requirements." 2018 EManufacturing \& Design Collaboration Symposium (Emdc 2018).

36. Williams, A. D. and A. H. Vette (2019). "A Vibrotactile Feedback Device for Seated Balance Assessment and Training." Jove-Journal of Visualized Experiments(143).

37. Zhao, Y. X., et al. (2017). "A Real-Time Bicycle Record System of Ground Conditions Based on Internet of Things." Ieee Access 5: 17525-17533.

38. Zhou, X. H., et al. (2019). "Underlay Drone Cell for Temporary Events: Impact of Drone Height and Aerial Channel Environments." Ieee Internet of Things Journal 6(2): 1704-1718.

\subsubsection{Spor Eğitimi ve Yetenek Gelişimi}

1. Arbour-Nicitopoulos, K. P., et al. (2018). "A Scoping Review of Inclusive Out-of-School Time Physical Activity Programs for Children and Youth With Physical Disabilities." Adapted Physical Activity Quarterly 35(1): 111-138.

2. Barricelli, B. R., et al. (2020). "Human Digital Twin for Fitness Management." 8: 26637-26664.

3. Baudisch, P., et al. (2014). Imaginary reality basketball: A ball game without a ball. CHI'14 Extended Abstracts on Human Factors in Computing Systems: 575-578.

4. Bernardina, G. R. D., et al. (2019). "Moving system with action sport cameras: 3D kinematics of the walking and running in a large volume." PLoS One 14(11).

5. Collins, D. and L. Collins (2020). "Developing coaches' professional judgement and decision making: Using the 'Big 5'." Journal of Sports Sciences.

6. Haijun, B. and G. J. J. o. H. B. A. U. Yunli (2013). "Applied Research on Computer Virtual Reality Technology in College Physical Training []." 3. 

denemesi. Journal of Human Sciences, 17(4), 1149-1176. doi:10.14687/jhs.v17i4.6088

7. Kiefer, A. W., et al. (2017). "Virtual Reality As a Training Tool to Treat Physical Inactivity in Children." Frontiers in Public Health 5.

8. Kohe, G. Z. and L. G. Purdy (2019). "Analytical attractions and the techno-continuum: Conceptualising data obsessions and consequences in elite sport." Sport Education and Society 24(7): 742-755.

9. Kong, F. and Y. M. Wang (2019). "Design of computer interactive system for sports training based on artificial intelligence and improved support vector." Journal of Intelligent \& Fuzzy Systems 37(5): 6165

10. Nie, Z. C. (2020). "Research on sports planning and stability control of humanoid robot table tennis." International Journal of Advanced Robotic Systems 17(1).

11. Rajsp, A. and I. Fister (2020). "A Systematic Literature Review of Intelligent Data Analysis Methods for Smart Sport Training." Applied Sciences-Basel 10(9).

12. Reeves, M. J. and S. J. Roberts (2020). "A bioecological perspective on talent identification in junior-elite soccer: A Pan-European perspective." Journal of Sports Sciences 38(11-12): 1259-1268.

13. Rein, R., et al. (2017). "'Which pass is better?" Novel approaches to assess passing effectiveness in elite soccer." Human Movement Science 55: 172-181.

14. Sajjad, M., et al. (2020). "Human Behavior Understanding in Big Multimedia Data Using CNN based Facial Expression Recognition." Mobile Networks \& Applications 25(4): 16111621.

15. Sands, W. A., et al. (2017). "Modern Techniques and Technologies Applied to Training and Performance Monitoring." International Journal of Sports Physiology and Performance 12: 63-72.

16. Santos, O. C. (2019). "Artificial Intelligence in Psychomotor Learning: Modeling Human Motion from Inertial Sensor Data." International Journal on Artificial Intelligence Tools 28(4).

17. Saxon, L., et al. (2020). "Continuous Measurement of Reconnaissance Marines in Training With Custom Smartphone App and Watch: Observational Cohort Study." Jmir Mhealth and Uhealth 8(6).

18. Sigrist, R., et al. (2015). "Sonification and haptic feedback in addition to visual feedback enhances complex motor task learning." Experimental Brain Research 233(3): 909-925.

19. Tian, G., et al. (2019). "Rich lamellar crystal baklava-structured PZT/PVDF piezoelectric sensor toward individual table tennis training." Nano Energy 59: 574-581.

20. Tuncer, T., et al. (2020). "Ensemble residual network-based gender and activity recognition method with signals." Journal of Supercomputing 76(3): 2119-2138.

21. Wang, Y., et al. (2018). "IoT for next-generation racket sports training." 5(6): 4558-4566.

22. Witkowski, K., et al. (2016). "The use of augmented-reality technology to improve judo techniques. Premises, assumptions, methodology, research tools, preliminary scenarios - the first stage of the study." Archives of Budo 12: 355-367.

23. Xiong, X. W. (2020). "Study on the intelligent system of sports culture centers by combining machine learning with big data." Personal and Ubiquitous Computing 24(1): 151-163.

24. Zheng, W. and A. Wu (2020). "Research on the Performance of Big Data Decision Convolutional Neural Network (CNN) in Sports Public Service." Basic \& Clinical Pharmacology \& Toxicology 126: 195-196.

25. Yin, J. and X. Q. Wang (2019). "Study on safety mode of dragon boat sports physical fitness training based on machine learning." Safety Science 120: 1-5. 
Ünlü, Y., \& Taş, Z. (2020). Sporda endüstri 4.0 uygulama alanları kapsamında yapılan araştırmalar üzerine bir bibliyografya denemesi. Journal of Human Sciences, 17(4), 1149-1176. doi:10.14687/jhs.v17i4.6088

\subsubsection{Spor Sakatlıkları ve Sporcu Sağlı̆̆1}

1. Belcher, S., et al. (2020). "Ten-year nationwide review of netball ankle and knee injuries in New Zealand." Journal of Science and Medicine in Sport 23(10): 937-942.

2. De Vita, M., et al. (2015). "Epidemiological study on artistic gymnastics injuries." Medicina Dello Sport 68(3): 487-495.

3. Heim, D., et al. (2014). "A 13-year analysis from Switzerland of non-fatal sledging (sledding or tobogganing) injuries." Injury-International Journal of the Care of the Injured 45(1): 338341.

4. Hui, Q. L. (2019). "Prevention and Treatment of Occupational Diseases of Ocean Seafarers by Sports Based on Big Data." Journal of Coastal Research: 773-777.

5. Irgens, A., et al. (2016). "Diving exposure and health effects in divers working in different areas of professional diving." Int Marit Health 67(4): 235-242.

6. Jones, B. H. and V. D. Hauschild (2015). "Physical Training, Fitness, and Injuries: Lessons Learned from Military Studies." Journal of Strength and Conditioning Research 29: S57-S64.

7. Kapinski, N., et al. (2019). "Monitoring of the Achilles tendon healing process: can artificial intelligence be helpful?" Acta of Bioengineering and Biomechanics 21(1): 103-111.

8. Nayak, S. P., et al. (2019). "SIMAS: smart IoT model for acute stroke avoidance." International Journal of Sensor Networks 30(2): 83-92.

9. Niederer, D., et al. (2018). "Return to play, performance, and career duration after anterior cruciate ligament rupture: A case-control study in the five biggest football nations in Europe." Scandinavian Journal of Medicine \& Science in Sports 28(10): 2226-2233.

10. Parker, W. and B. B. Forster (2019). "Artificial intelligence in sports medicine radiology: what's coming?" British Journal of Sports Medicine 53(19): 1201-1202.

11. Sikka, R. S., et al. (2019). "Analytics in Sports Medicine Implications and Responsibilities That Accompany the Era of Big Data." Journal of Bone and Joint Surgery-American Volume 101(3): 276-283.

12. Stevenson, J. M. (2014). "Looking forward by looking back: Helping to reduce work-related musculoskeletal disorders." Work 47(1): 137-141.

13. Su, Y. F. (2019). "Implementation and Rehabilitation Application of Sports Medical Deep Learning Model Driven by Big Data." Ieee Access 7: 156338-156348.

14. Subasi, A., et al. (2018). IoT based mobile healthcare system for human activity recognition. 2018 15th Learning and Technology Conference (L\&T), IEEE.

15. Tanisawa, K., et al. (2020). "Sport and exercise genomics: the FIMS 2019 consensus statement update." British Journal of Sports Medicine 54(16): 969-975.

16. Wan, M. M., et al. (2019). "A Safe and Efficient Strategy for the Rapid Elimination of Blood Lead In Vivo Based on a Capture-Fix-Separate Mechanism." Angew Chem Int Ed Engl 58(31): 10582-10586.

17. Wasserstein, D. and U. Sheth (2018). "Administrative Databases in Sports Medicine Research." Clinics in Sports Medicine 37(3): 483-+.

18. Wilkerson, G. B., et al. (2018). "Mitigating sports injury risks using internet of things and analytics approaches." 38(7): 1348-1360.

19. Wiszomirska, I., et al. (2017). "The impact of sensorimotor training on postural stability and motor skills of basketball players in the prevention of injuries." Medicina Dello Sport 70(3): 354-364.

20. Curran-Sills, G. (2018). "Safety in Mixed Martial Arts: a 7-Year Review of Cancelled MMA Bouts in Calgary, Alberta, During the Pre-bout Medical Examination Period." Sports Medicine-Open 4. 

denemesi. Journal of Human Sciences, 17(4), 1149-1176. doi:10.14687/jhs.v17i4.6088

\subsubsection{Spor Tesisleri}

1. Adams, K., et al. (2020). "From the field of play to the laboratory: Recreating the demands of competition with augmented reality simulated sport." Journal of Sports Sciences 38(5): 486-493.

2. Al Rashid, A., et al. (2020). "Utilization of Banana Fiber-Reinforced Hybrid Composites in the Sports Industry." Materials (Basel) 13(14).

3. Citraro, L., et al. (2020). "Real-time camera pose estimation for sports fields." Machine Vision and Applications 31(3).

4. Fenil, E., et al. (2019). "Real time violence detection framework for football stadium comprising of big data analysis and deep learning through bidirectional LSTM." 151: 191200.

5. Gao, X. A. (2019). "Ecological Research on Application of Intelligent Management System Based on Internet of Things in University Feather Stadium in Gansu Province." Ekoloji 28(107): 4819-4825.

6. Kazan, H., et al. (2015). "Economic Development of Ski Industry in Experimental Innovation: Example of Palandoken Turkey and Alps Switzerland." World Conference on Technology, Innovation and Entrepreneurship: 487-492.

7. Kubler, S., et al. (2017). "Open IoT Ecosystem for Sporting Event Management." Ieee Access 5: 7064-7079.

8. Kumar, N. and N. Sukavanam (2020). "A weakly supervised CNN model for spatial localization of human activities in unconstraint environment." Signal Image and Video Processing 14(5): 1009-1016.

9. Liu, T., et al. (2016). "Model for evaluating the management performance of the sport grounds with interval-valued intuitionistic uncertain linguistic information." Journal of Intelligent \& Fuzzy Systems 31(3): 1535-1544.

10. Monteiro, L. G., et al. (2017). "One-Year Monitoring PV Power Plant Installed on Rooftop of MineirAo Fifa World Cup/Olympics Football Stadium." Energies 10(2).

11. Mosleh, Y., et al. (2018). "Designing safer composite helmets to reduce rotational accelerations during oblique impacts." Proc Inst Mech Eng H 232(5): 479-491.

12. Municio, E., et al. (2019). "Continuous Athlete Monitoring in Challenging Cycling Environments Using IoT Technologiesis." Ieee Internet of Things Journal 6(6): 1087510887.

13. O'Brolchain, F., et al. (2019). "The Ethics of Smart Stadia: A Stakeholder Analysis of the Croke Park Project." Science and Engineering Ethics 25(3): 737-769.

14. Samuel, R. D. J., et al. (2019). "Real time violence detection framework for football stadium comprising of big data analysis and deep learning through bidirectional LSTM." Computer Networks 151: 191-200.

15. Suganuma, T., et al. (2018). "Multiagent-Based Flexible Edge Computing Architecture for IoT." Ieee Network 32(1): 16-23.

16. Tay, S. P., et al. (2017). "Skin friction related behaviour of artificial turf systems." J Sports Sci 35(15): 1500-1507.

17. Watterson, A. (2017). "Artificial Turf: Contested Terrains for Precautionary Public Health with Particular Reference to Europe?" International Journal of Environmental Research and Public Health 14(9). 
Ünlü, Y., \& Taş, Z. (2020). Sporda endüstri 4.0 uygulama alanları kapsamında yapılan araştırmalar üzerine bir bibliyografya denemesi. Journal of Human Sciences, 17(4), 1149-1176. doi:10.14687/jhs.v17i4.6088

\section{Yöntem}

Bu çalışma sporda endüstri 4.0 kapsamında yapılan bibliyografyaları içermektedir. Bu amaçla konu ile ilgili araştırma makaleleri, bilimsel bildiriler, kitap, yüksek lisans ve doktora çalışmaları konu bazlı derinlemesine incelenmiştir. EndNote 9 paket programının kullanıldığı çalışmada 2015- 2020 yilları arasinda Web of Science, Web of Sciences Core, PubMed, LISTA ve Google Schoolar veri tabanları taranarak 249 çalışmanın tam künyeleri verilmiştir. Çalışmalar; giyilebilir akıllı cihazlar, performans ve analiz, tahmin tabanlı uygulama ve programlar, spor malzeme ve materyalleri, spor eğitimi ve yetenek gelişimi, spor sakatlıkları ve sporcu sağlığ1 ve spor tesisleri başlıkları altında kategorize edilerek listelenmiştir.

\section{Sonuç}

Bu çalışmada sporda endüstri 4.0 kapsamında yayınlanan araştırmalar incelenmiştir. Sporda endüstri 4.0 kapsamında yapılan çalışmaların şu konularda yoğunlaştığı görülmektedir:

- Sporcu performansını geliştirme ve bu performansa bağlı analizler için üretilen uygulamalar,

- Sporcu eğitimi ve yetenek gelişimine yönelik üretilen uygulamalar,

- Tahmin tabanli uygulama ve programlar,

- Giyilebilir akıllı cihazlar, spor malzeme ve materyalleri,

- Spor sakatlıklarını önleme ve sporcu sağlığını korumaya yönelik uygulamalar,

- Spor tesislerinin fonksiyonlarını arttırmaya yönelik yapılan uygulamalardır.

Konu bağlamındaki araştırmalar incelendiğinde sporcu performansını arttırmaya yönelik ve bu performansın analizi için gerekli uygulamalar, akıllı sistemler, cihazlar ve programların öne çıktı̆ı görülmektedir. Sporun çeşitli branşlarında kullanılan giyilebilir akıllı cihazlar da yine sporda endüstri 4.0 kapsamında yoğun olarak ele alınan çalışma konuları olmuştur. Alanla ilgili spor malzeme ve materyallerin üretiminin özellikle gelişmiş ülkelerde hızla yaygınlaştığı, spor sakatlıkları ve sporcu sağlığ gibi hususlarda endüstri 4.0 teknolojisinden azami derecede istifade edildiği tespit edilmiştir. Çalışmaların yoğunlaştı̆̆ bir başka konu ise sportif faaliyetleri toplu olarak izleyenlerin bulunduğu ve onlara her açıdan daha konforlu anlar yaşatmayı amaçlayan spor tesisleri olmuştur.

$\mathrm{Bu}$ çalışmaların sporda özellikle big data teknolojisi ile veri ve analitiklerin entegrasyonu sağlanarak yapıldığı görülmektedir. Araştırmalar genellikle amatör ve profesyonel sporlarda deneyim ve yetenek gelişimi sağlama, performans ve rekabet avantajını arttırmaya yöneliktir. Araştırmalarda teknolojinin sağladığ1 yenilikçi araçların kullanımı ile kulüplerin, antrenörlerin, sporcuların; rakipleri ile ilgili stratejik hamle ve uygulamalarla daha avantajlı konuma geçmeleri hedeflenmektedir. Spordaki bu yenilikler ve gelişmeler doğrudan performans artısı sağlamaya yöneliktir. Alandaki teknolojik yenilikler ve gelişmeler son yıllarda profesyonel kulüp saysındaki artış, prestijli turnuva, organizasyonların çoğalması, spor ligleri ve spor bahis oyunlarına olan ilginin davasa şekilde büyümesine paralel olarak big data, yapay zeka, nesnelerin interneti gibi teknolojilerin kullanıldığ1 çalışmaların sayısında artışa neden olmaktadır. Bu çalışmaların, takım ve sporcuların antrenman yapma şekillerine, spor seyircisi ve taraftar etkileşimine daha önce tecrübe edilmemiş katkılar sağlamaktadır. IOT ve Big data ile ilgili olan çalısmaların sporcu eğitimi, sporcu tanıma ve yönlendirme, oyun içi strateji geliştirme, sporcu güvenliği ve sağllğı, akılll spor tesisi gibi alanlarda yoğunlaştığı görülmektedir. Buna karşı, Endüstri 4.0 kapsamındaki teknolojilerin spor faaliyetlerinin her alanında sağladığı konfor, kolaylık ve faydanın yanı sıra teknolojinin insan yaşamındaki diğer kullanım alanlarında olduğu gibi spor alanında da bazı tehditler içerebilmektedir. Bu tehditler sporla ilgili istihdam alanları başta olmak üzere özellikle Big data ve IOT teknolojilerinin spora özgü uygulamalarda elde edilen verilerin korunmas1, veri mahremiyeti, siber tehditler ve bu siber tehditlere karşı konulması hususlarında ortaya çıkmaktadır.

Sporda Endüstri 4.0 bileşenleri kapsamındaki araştırmalar gelişmiş ülkelerde birçok spor branşında yaygınlık kazanarak özellikle Big data ve IOT tabanlı teknolojilerin kullanımının öne çıktığını göstermektedir. Bu çalışmalar giyilebilir teknoloji, ergonomik spor malzeme ve materyalleri ile sporcu performansını ölçmeye ve bu performansın analiz edilmesine yönelik araştırmalarda 
Ünlü, Y., \& Taş, Z. (2020). Sporda endüstri 4.0 uygulama alanları kapsamında yapılan araştırmalar üzerine bir bibliyografya denemesi. Journal of Human Sciences, 17(4), 1149-1176. doi:10.14687/jhs.v17i4.6088

yoğunlaşmaktadır. Türkiye'de Endüstri 4.0 bileşenlerinin sağlık, savunma sanayii ve endüstri alanında yaygın olarak kullanılmasına rağmen spor alanındaki uygulamalarda henüz yaygınlaşmadığ1 görülmektedir. Bu durum Endüstri 4.0 teknolojilerinin spor alanındaki altyapısının Türkiye’de sağlıklı bir şekilde kurulmasının gerekliliğini öne çıkarmaktadır. Bu çalışmalar özellikle örgün eğitim müfredatına spor teknolojileri ve inovasyon derslerinin eklenmesi ve içeriğinin sürekli güncellenmesi ile gerçekleştirilebileceği düşünülmektedir. Aynıca Türkiye'de düzenlenen ulusal ve uluslararası bilim fuarlarında sporda teknoloji kullanımının önemine dikkat çeken araştırmalara daha fazla yer verilmelidir. Bu noktada devlete ait spor kurum ve kuruluşlarının özel sektörle tesis edeceği ar-ge birimleri eliyle sporda teknoloji kullanımına yönelik uygulamalanın takibi ve bu yöndeki projelerin geliştirilmesi çerçevesindeki desteklerin arttırılması gerekmektedir.

\section{Kaynakça}

Akpa, AH, Masashi Fujiwara, Hirohiko Suwa, Yutaka Arakawa, and Yasumoto. (2019). 'A smart glove to track fitness exercises by reading hand palm'.

Al-Makhadmeh, Zafer, and Amr. (2019) 'Utilizing IoT wearable medical device for heart disease prediction using higher order Boltzmann model: A classification approach', 147: 106815.

Al Rashid, A., M. Y. Khalid, R. Imran, U. Ali, and M. Koc. (2020). 'Utilization of Banana FiberReinforced Hybrid Composites in the Sports Industry', Materials (Basel), 13.

Ali, Usman, Arun Choudhary, and Mohd. (2018). International Journal of Applied Engineering Research Abbas. 'Conceptualization, Design and Development of the Football Bladder Making Machine under the concept of Industry 4.0', 13: 218-22.

Baheti, Radhakisan, and Helen. (2011). The impact of control technology Gill. 'Cyber-physical systems', 12: 161-66.

Barricelli, B. R., E. Casiraghi, J. Gliozzo, A. Petrini, and S. Valtolina. (2020). 'Human Digital Twin for Fitness Management', Ieee Access, 8: 26637-64.

Baudisch, Patrick, Henning Pohl, Stefanie Reinicke, Emilia Wittmers, Patrick Lühne, Marius Knaust, Sven Köhler, Patrick Schmidt, and Christian Holz. (2014). 'Imaginary reality basketball: A ball game without a ball.' in, CHI'14 Extended Abstracts on Human Factors in Computing Systems.

Behravan, I., S. H. Zahiri, S. M. Razavi, and R. Trasarti. (2019). 'Finding Roles of Players in Football Using Automatic Particle Swarm Optimization-Clustering Algorithm', Big Data, 7: 35-56.

Bradley, Justin M, and Ella, M. (2015). 'Optimization and control of cyber-physical vehicle systems', 15: 23020-49.

Caporaso, Teodorico, Matthew Worsey, Hugo G Espinosa, David V Thiel, Angela Palomba, Stanislao Grazioso, Dario Panariello, Giuseppe Di Gironimo, and Antonio Lanzotti. (2020). "A preliminary approach for swimming performance analysis of fisdir elite athletes with intellectual impairment using an inertial sensor." In 2020 IEEE International Workshop on Metrology for Industry 4.0 \& IoT, 116-20. IEEE.

Chen, Hsinchun, Roger HL Chiang, and Veda. (2012). 'Business intelligence and analytics: From big data to big impact': 1165-88.

Citraro, L., P. Marquez-Neila, S. Savare, V. Jayaram, C. Dubout, F. Renaut, A. Hasfura, H. Ben Shitrit, and P. Fua. (2020). 'Real-time camera pose estimation for sports fields', Machine Vision and Applications, 31.

Conti, Mauro, Ali Dehghantanha, Katrin Franke, and Steve Watson. (2018). "Internet of Things security and forensics: Challenges and opportunities." In.: Elsevier.

Dei, M., J. Aymerich, M. Piotto, P. Bruschi, F. J. del Campo, and F. Serra-Graells. (2019). 'CMOS Interfaces for Internet-of-Wearables Electrochemical Sensors: Trends and Challenges', Electronics, 8.

Dmonte, Ruth, and Asher Dmello. (2017). 'Big Data in Sports', International Journal of Engineering Research \& Technology (IJERT). 
Ünlü, Y., \& Taş, Z. (2020). Sporda endüstri 4.0 uygulama alanları kapsamında yapılan araştırmalar üzerine bir bibliyografya denemesi. Journal of Human Sciences, 17(4), 1149-1176. doi:10.14687/jhs.v17i4.6088

Drath, Rainer, and Alexander \%J IEEE industrial electronics magazine Horch. (2014). 'Industrie 4.0: Hit or hype?[industry forum]', 8: 56-58.

Duncan, Olly, Todd Shepherd, Charlotte Moroney, Leon Foster, Praburaj D Venkatraman, Keith Winwood, Tom Allen, and Andrew (2018). 'Review of auxetic materials for sports applications: Expanding options in comfort and protection', 8: 941.

Fenil, E, Gunasekaran Manogaran, GN Vivekananda, T Thanjaivadivel, S Jeeva, and Ahilan. (2019). 'Real time violence detection framework for football stadium comprising of big data analysis and deep learning through bidirectional LSTM', 151: 191-200.

Fialho, Gabriel, Aline Manhães, and João Paulo \%J Procedia Computer Science Teixeira. (2019). 'Predicting Sports Results with Artificial Intelligence-A Proposal Framework for Soccer Games', 164: 131-36.

Goes, FR, LA Meerhoff, MJO Bueno, DM Rodrigues, FA Moura, MS Brink, MT Elferink-Gemser, AJ Knobbe, SA Cunha, and RS, Torres. (2020). 'Unlocking the potential of big data to support tactical performance analysis in professional soccer: A systematic review': 1-16.

Haijun, Bai, and Gao \%J Journal of Heilongjiang Bayi Agricultural University Yunli. (2013). 'Applied Research on Computer Virtual Reality Technology in College Physical Training [D]', 3 .

Hassan, A., A. R. Akl, I. Hassan, and C. Sunderland. (2020). 'Predicting Wins, Losses and Attributes' Sensitivities in the Soccer World Cup 2018 Using Neural Network Analysis', Sensors, 20.

Hermann, Mario, Tobias Pentek, and Boris Otto. (2016). "Design principles for industrie 4.0 scenarios." In 2016 49th Hawaii international conference on system sciences (HICSS), 3928-37. IEEE.

İçten, Tarık, and Güngör. (2017). Gazi Üniversitesi Fen Bilimleri Dergisi Part C: Tasarım ve Teknoloji Güngör. 'Artırılmış gerçeklik üzerine son gelişmelerin ve uygulamaların incelenmesi', 5: 111-36.

Itoh, Yuta, Jason Orlosky, Kiyoshi Kiyokawa, and Gudrun Klinker. (2016). "Laplacian vision: Augmenting motion prediction via optical see-through head-mounted displays." In Proceedings of the 7th Augmented Human International Conference, 1-8.

James, Daniel A, and Nicola Petrone. (2016). Sensors and Wearable Technologies in Sport: Technologies, Trends and Approaches for Implementation (Springer).

Kagermann, Henning, Wolf-Dieter Lukas, and Wahlster. (2011). 'Industrie 4.0: Mit dem Internet der Dinge auf dem Weg zur 4. industriellen Revolution', 13.

Kajastila, R., L. Holsti, and P. Hamalainen. (2016). 'The Augmented Climbing Wall: High-Exertion Proximity Interaction on a Wall-Sized Interactive Surface', 34th Annual Chi Conference on Human Factors in Computing Systems, Chi 2016: 758-69.

Keshtkar Langaroudi, Milad, Mohammadreza. (2019). 'Sports result prediction based on machine learning and computational intelligence approaches: A survey', 5: 27-36.

Lapadula, Valerio, Anna Sabatini, Alessandro Zompanti, Silvia Buscaglione, Davide Lanaro, and Mario Meron. (2020). "A Body Hydration Analysis System to improve running performance." In 2020 IEEE International Workshop on Metrology for Industry 4.0 \& IoT, $507-$ 10. IEEE.

Liu, J. W., S. H. Chen, Y. C. Huang, and C. T. Wang. (2020). 'An Intelligent Identification Model for the Selection of Elite Rowers by Incorporating Internet-of-Things Technology', Ieee Access, 8: 31234-44.

Makridakis, Spyros. (2017). 'The forthcoming Artificial Intelligence (AI) revolution: Its impact on society and firms', 90: 46-60.

Marques-Deak, Andrea, Giovanni Cizza, Farideh Eskandari, Sara Torvik, Israel C Christie, Esther M Sternberg, Terry M Phillips, (2006). 'Measurement of cytokines in sweat patches and plasma in healthy women: validation in a controlled study', 315: 99-109. 
Ünlü, Y., \& Taş, Z. (2020). Sporda endüstri 4.0 uygulama alanları kapsamında yapılan araştırmalar üzerine bir bibliyografya denemesi. Journal of Human Sciences, 17(4), 1149-1176. doi:10.14687/jhs.v17i4.6088

Massaroni, Carlo, Andrea Nicolò, Daniela Lo Presti, Massimo Sacchetti, and Emiliano Schena. (2020). "Respiratory monitoring during cycling exercise: performance assessment of a smart t-shirt embedding fiber optic sensors." In 2020 IEEE International Workshop on Metrology for Industry 4.0 \& IoT, 49-53. IEEE.

McCaffery, Fergal, Özden Özcan-Top, Ceara Treacy, Pangkaj Paul, John Loane, Jennifer Crilly, and Arthur Mc Mahon. (2018). "A process framework combining safety and security in practice." In European Conference on Software Process Improvement, 173-80. Springer.

Mueller, Egon, Xiao-Li Chen, and Ralph. (2017). 'Challenges and requirements for the application of industry 4.0: a special insight with the usage of cyber-physical system', 30: 1050-57.

Nadikattu, Rahul Reddy \%J Journal of Xidian University. (2020). 'Implementation of New Ways of Artificial Intelligence in Sports', 14: 5983-97.

Neugebauer, Reimund, Sophie Hippmann, Miriam Leis, and Martin Landherr. (2016). "Industrie 4.0-From the perspective of applied research." In.: Elsevier.

Padmapriya, A, P. Subhasri, (2013). 'Cloud computing: security challenges and encryption practices', 3 .

Pappalardo, L., P. Cintia, P. Ferragina, E. Massucco, D. Pedreschi, and F. Giannotti. 2019. 'PlayeRank: Data-driven Performance Evaluation and Player Ranking in Soccer via a Machine Learning Approach', Acm Transactions on Intelligent Systems and Tecbnology, 10.

Rein, R., and D. Memmert. (2016). 'Big data and tactical analysis in elite soccer: future challenges and opportunities for sports science', Springerplus, 5.

Resta, Paolo, Daniela Lo Presti, Emiliano Schena, Carlo Massaroni, Domenico Formica, Taesung Kim, and Donjoo Shin. (2020). "A wearable system for knee flexion/extension monitoring: design and assessment." In 2020 IEEE International Workshop on Metrology for Industry 4.0 \& IoT, 273-77. IEEE.

Rotshtein, Alexander P, Morton and Posner, (2005). 'Football predictions based on a fuzzy model with genetic and neural tuning', 41: 619-30.

Schumachera, Andreas, Selim Erolb, and Wilfried Sihna. (2016). 'A maturity model for assessing Industry 4.0 readiness and maturity of manufacturing enterprises'.

Schwab, Klaus. (2019). Dördüncü Sanayi Devrimini Şekillendirmek (Optimist Yayın Grubu).

Shaw, Jesse, Flavio Villanustre, Borko Furht, Ankur Agarwal, and Abhishek Jain. (2016). 'Modeling Ebola Spread and Using HPCC/KEL System.' in, Big Data Technologies and Applications (Springer).

Smyth, Carol ME, Samantha L Winter, and John W Dickinson. (2020). "Optoelectronic plethysmography derived breathing parameters can differ between athletes with and without a dysfunctional breathing pattern during exercise." In 2020 IEEE International Workshop on Metrology for Industry 4.0 \& IoT, 54-58. IEEE.

Sourin, Alexei. (2017). 'Case study: shared virtual and augmented environments for creative applications.' in, Research and development in the academy, creative industries and applications (Springer).

Subasi, Abdulhamit, Mariam Radhwan, Rabea Kurdi, and Kholoud Khateeb. (2018). "IoT based mobile healthcare system for human activity recognition." In 2018 15th Learning and Technology Conference (L\&T), 29-34. IEEE.

Subramanian, Nalini and Andrews. (2018). 'Recent security challenges in cloud computing', 71: 2842.

T. Caporaso, S.Grazioso, D. Panariello, G. Di Gironimo, and A. Lanzotti. 2020. 'A wearable inertial device based on biomechanical parameters for sports performance analysis in race-walking: preliminary results'.

Toft, Anders Dyhr, Lars Bjørn Jensen, Helle Bruunsgaard, Tobias Ibfelt, Jens Halkjær-Kristensen, Mark Febbraio, and Bente Klarlund Pedersen. (2002). 'Cytokine response to eccentric exercise in young and elderly humans', 283: C289-C95. 
Ünlü, Y., \& Taş, Z. (2020). Sporda endüstri 4.0 uygulama alanları kapsamında yapılan araştırmalar üzerine bir bibliyografya denemesi. Journal of Human Sciences, 17(4), 1149-1176. doi:10.14687/jhs.v17i4.6088

Vermesan, Ovidiu, and Peter Friess. (2013). Internet of things: converging technologies for smart environments and integrated ecosystems (River publishers).

Wang, Yufan, Meng Chen, Xinyu Wang, Rosa HM Chan, and Wen, Li. (2018). 'IoT for nextgeneration racket sports training', 5: 4558-66.

Wiehr, Frederik, Felix Kosmalla, Florian Daiber, and Antonio Krüger. (2016). "betaCube: Enhancing training for climbing by a self-calibrating camera-projection unit." In Proceedings of the 2016 CHI Conference Extended Abstracts on Human Factors in Computing Systems, 19982004.

Wilkerson, G. B., A. Gupta, and M. A. Colston. (2018). 'Mitigating Sports Injury Risks Using Internet of Things and Analytics Approaches', Risk Analysis, 38: 1348-60.

Xia, Kun, Hanyu Wang, Menghan Xu, Zheng Li, Sheng He, and Yusong (2020). 'Racquet Sports Recognition Using a Hybrid Clustering Model Learned from Integrated Wearable Sensor', 20: 1638.

Xiong, Jian, Liguo Lu, Hengbing Wang, Jie Yang, and Guan (2019). 'Object-Level Trajectories Based Fine-Grained Action Recognition in Visual IoT Applications', 7: 103629-38.

Yu, Hongying (2020). 'Research and optimization of sports injury medical system under the background of Internet of things': e3929.

Yu, L. (2018a). 'Cloud storage-based personalized sports activity management in Internet plus O2O sports community', Concurrency and Computation-Practice \& Experience, 30.

Yu, L. (2018b). 'Sports activity detection, organization and evaluation in online to offline sports community', Cognitive Systems Research, 52: 785-92.

\section{Extended English Summary}

\section{Aim}

Developed countries develop their production sites within the scope of industry 4.0 technology components and experience constant change and transformation to establish economic superiority. This situation allows them to produce more in various fields and thus to rise to a more advantageous position economically. Industry 4.0 technology affects areas within the scope of the sports industry such as sports tourism, athlete performance, athlete health, sports publishing, sports textile products, sports education and training, sports management and human resources, and creates an international competition environment in terms of production and performance. In this study, it is aimed to examine the researches about the usage areas of industry 4.0 in sports. From this point on, researches in the context of the subject have been presented with bibliographic method. In the conclusion section, the weaknesses and possibilities of youth sociology were discussed, and efforts were made to present a projection on what to do about the field. In this respect, a youth sociology evaluation has been tried to be made on the prominent topics, forgotten aspects and themes left incomplete in youth sociology studies.

\section{Methodology}

This study includes bibliographies made within the scope of industry 4.0 in sports. For this purpose, research articles, scientific reports, books, master's and doctoral studies on the subject were examined in depth. In the study, in which EndNote 9 package program was used, the full data of 249 studies were given by scanning Web of Science, Web of Sciences Core, PubMed, LISTA and Google Schoolar databases between 2015-2020. Studies; Wearable smart devices are categorized and listed under the headings of performance and analysis, prediction-based applications and programs, sports equipment and materials, sports training and talent development, sports injuries and athlete health and sports facilities.

\section{Results}

In this study, researches published within the scope of industry 4.0 in sports were examined. It is seen that the studies conducted within the scope of industry 4.0 in sports focus on: Applications produced for improving athlete performance and analysis based on this performance, 

denemesi. Journal of Human Sciences, 17(4), 1149-1176. doi:10.14687/jhs.v17i4.6088

Applications produced for athlete training and talent development, Prediction based applications and programs, Wearable smart devices, sports equipment and materials, Practices for preventing sports injuries and protecting athlete health, These are applications to increase the functions of sports facilities.

When the researches in the context of the subject are examined, it is seen that the applications, smart systems, devices and programs that aim to increase the performance of athletes and which are necessary for the analysis of this performance come to the fore. Wearable smart devices used in various branches of sports have also been the subject of intensive study within the scope of industry 4.0 in sports. It has been determined that the production of sports equipment and materials related to the field has become widespread especially in developed countries, and the industry 4.0 technology has been utilized to the maximum in matters such as sports injuries and athlete health. Another focus of the studies was the sports facilities, where people watch sports activities collectively and aimed to provide them with more comfortable moments in every aspect.

\section{Discussion}

It is seen that these studies are carried out by integrating data and analytics especially with big data technology in sports. Research is generally aimed at providing experience and talent development, performance and competitive advantage in amateur and professional sports. With the use of innovative tools provided by technology in researches, clubs, trainers, athletes; It is aimed for them to reach a more advantageous position with strategic moves and practices regarding their competitors. These innovations and developments in sports are directly aimed at increasing performance. Technological innovations and developments in the field have led to an increase in the number of studies using technologies such as big data, artificial intelligence, internet of things, in parallel with the increase in the number of professional clubs, the proliferation of prestigious tournaments, organizations, and the growing interest in sports leagues and sports betting games. These studies provide unprecedented contributions to the way teams and athletes train, as well as to the interaction between sports spectators and fans. It is seen that studies related to IOT and Big data focus on athlete training, athlete recognition and guidance, in-game strategy development, athlete safety and health, and smart sports facility. On the other hand, in addition to the comfort, convenience and benefit provided by the technologies within the scope of Industry 4.0 in all areas of sports activities, the technology may also contain some threats in the field of sports as in other areas of use of the technology in human life. These threats arise especially in the fields of employment related to sports, especially the protection of data obtained in sports-specific applications of Big data and IOT technologies, data privacy, cyber threats and the protection of these cyber threats.

\section{Conclusion}

Research within the scope of Industry 4.0 components in sports has become widespread in many sports branches in developed countries and shows that the use of Big data and IOT-based technologies has become prominent. These studies focus on wearable technology, ergonomic sports materials and materials, and research on measuring athlete performance and analyzing this performance. 4.0 component of industrial health in Turkey, although widely used in industrial applications in the defense industry and sports fields is not yet widespread. In this case 4.0 technology infrastructure industries in Turkey in the area of sport highlights the necessity to build a healthy way. It is thought that these studies can be realized by adding sports technologies and innovation courses to the formal education curriculum and constantly updating their content. In addition, the research draws attention to the importance of using technology in sport in national and international science fairs held in Turkey should be given more space. At this point, it is necessary to increase the support for the follow-up of the applications for the use of technology in sports and the development of the projects in this direction by the $\mathrm{R} \& \mathrm{D}$ units that the state-owned sports institutions and organizations will establish with the private sector. 